\title{
Designs and applications of circularly polarized thermally activated delayed fluorescence molecules
}

Lucas Frédéric, Alaric Desmarchelier, Ludovic Favereau*, Grégory Pieters*

Dr. L. Frédéric, Dr. A. Desmarchelier, Dr. G. Pieters

Université Paris-Saclay, SCBM, CEA Paris Saclay

E-mail: gregory.pieters@cea.fr

Dr. L. Favereau, Université de Rennes, CNRS, ISCR - UMR 6226, 35042 Rennes, France E-mail: ludovic.favereau@univ-rennes1.fr

Keywords: Thermally Activated Delayed Fluorescence, Circularly Polarized Luminescence, Organic Light Emitting Diode

\section{Abstract:}

The design of fluorophores merging Circularly Polarized Luminescence (CPL) and Thermally Activated Delayed Fluorescence (TADF) properties has recently emerged as a promising direction for the development of efficient CP-Organic Light-Emitting Diodes (CP-OLEDs). This progress report gives an overview of the molecular designs explored to obtain CP-TADF properties, of their performances as chiral emitters in CP-OLEDs and discusses future challenges for this burgeoning field of research.

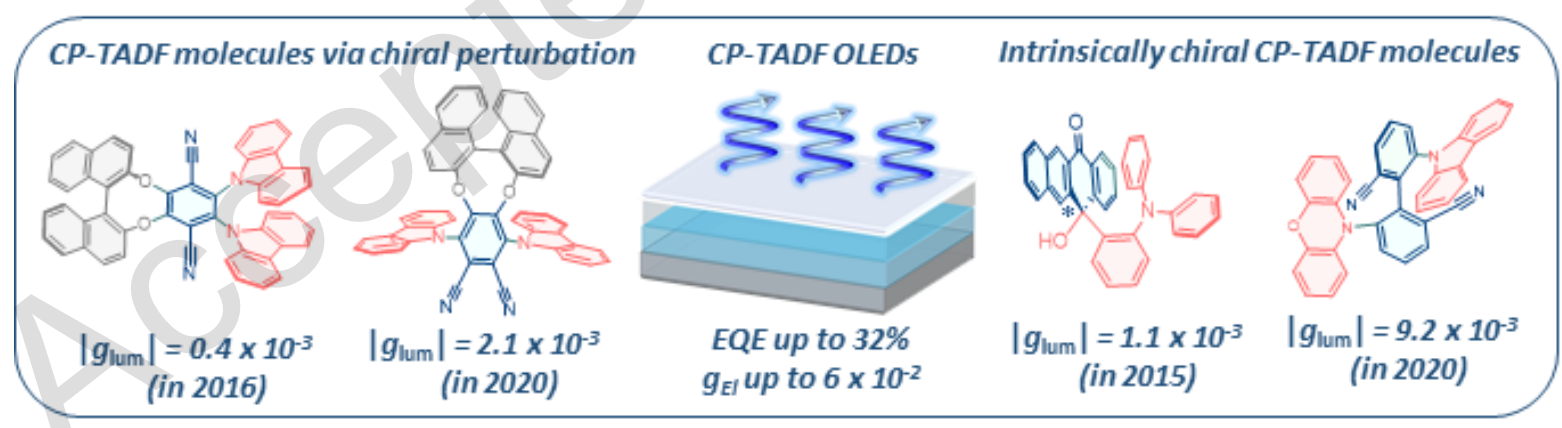




\section{Introduction}

Since the pioneering work of Tang and Van Slyke in the late 1980s, organic light-emitting diodes (OLEDs, figure 1A) have emerged as one of the most promising technologies for both lighting and display applications. ${ }^{[1]}$ Regarding the latter, compared to other light emitting devices, OLEDs can offer: a lower power consumption with improved image quality (including better contrast and higher brightness), a fuller viewing angle and faster refresh rates, and the added benefit of potential ultra-thin, flexible or even transparent screens. Schematically, in an OLED, holes and electrons, respectively generated by the cathode and the anode, recombine in the emissive layer to form excitons, which can produce photons via the electroluminescence process (see Figure 1B). ${ }^{[1]}$

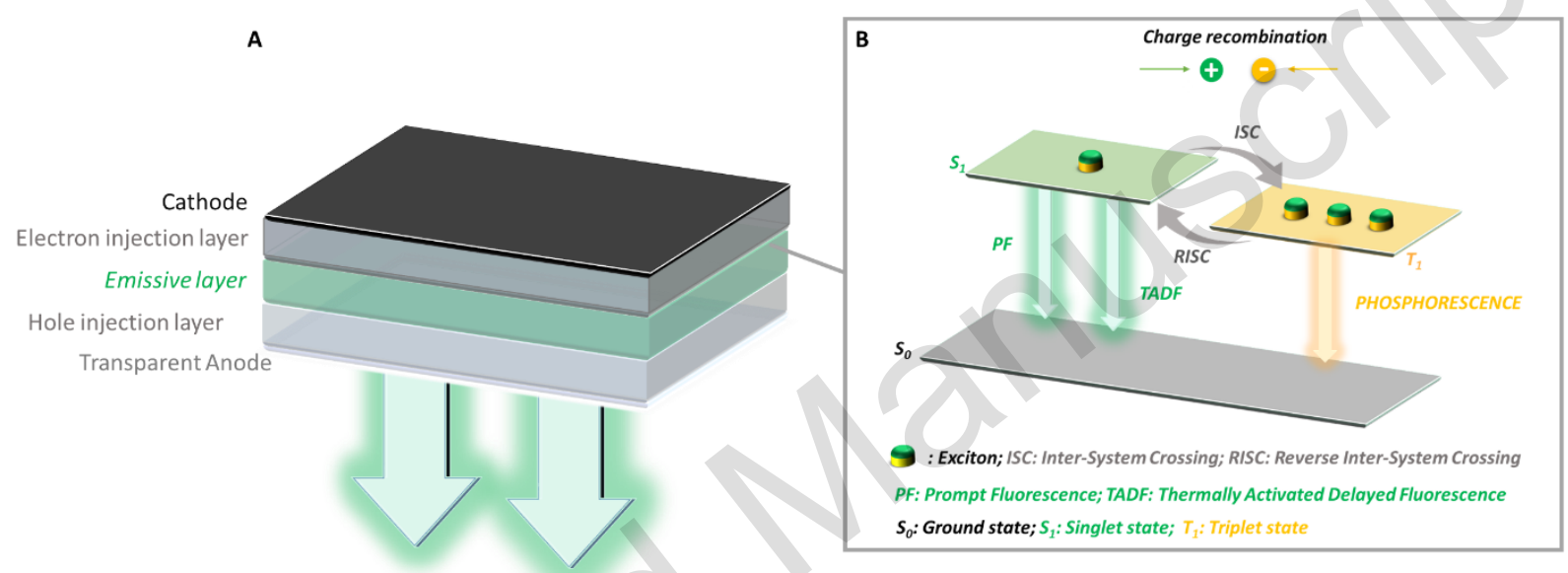

Figure 1: Simplified OLED stack and Jablonski Diagram with a emphasis on the thermally activated delayed fluorescence process

The efficiency of OLEDs can be evaluated notably by the determination of: 1) the Internal Quantum Efficiency (IQE), the proportion of all electron-hole recombinations in the emissive layer leading to the production of photons or 2) External Quantum Efficiency (EQE), the ratio of the number of photons emitted from the OLED to the number of electrons passing through the device. In the emissive layer, due to spin statistics, $25 \%$ of the excitons formed are in the singlet state and $75 \%$ are in the triplet state (Figure 1B). For this reason, the IQE of OLED can only reach $25 \%$ when emitters display only prompt fluorescence (which can only harvest energy from their singlet state). In the late 90s, to overcome this limitation, Baldo and Forrest have introduced the use of phosphorescent emitters permitting the harvest of triplet excitons to produce light and reach much higher IQE values (up to $100 \%$ considering the possible internal system crossing (ISC, Figure 1B) between $S_{1}$ and $T_{1}$ states). ${ }^{[2]}$ While such a strategy has resulted in high device performances and is currently the leading approach in OLED display applications, 
the use of rare and expensive metals remains prejudicial both ecologically and geopolitically due to the impact of noble metals exploitation. Accordingly, intense research programs are continuously undertaken to design and optimize the performances of other types of emitters. Among those investigative avenues, thermally activated delayed fluorescence (TADF) is one of the promising mechanisms by which performances could be drastically improved. ${ }^{[3]}$ This booming area of research has already been the subject of many articles, and detailed explanations about the molecular design principles, performances and applications of such materials can be found in dedicated recent reviews. ${ }^{[4]}$ Briefly, in TADF emitters, both singlet and triplet excitons can be harvested to produce light due to a small energy gap between their singlet and triplet excited states $\left(\Delta \mathrm{E}_{\mathrm{ST}}<0.5 \mathrm{eV}\right)$. The prerequisite molecular design to reach this condition while keeping strong oscillator strengths and luminescence efficiency involves mainly 3 different strategies (Figure 2): 1) Inducing an important steric hindrance in donoracceptor molecules to obtain a twisted intramolecular charge transfer (TICT) state; 2) Allowing a through-space charge transfer (TSCT) by controlling the distance between a donor and acceptor unit and their relative orientation; 3) Taking advantage of the multiple resonance effects in heteroaromatic compounds (e.g. nitrogen- and boron-containing molecules). ${ }^{[5]}$

1) TICT molecules

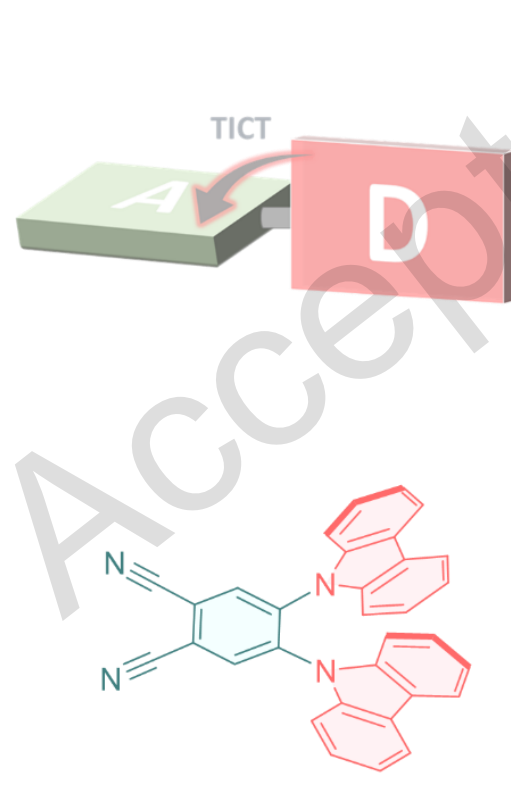

2CzPN
2) TSCT molecules
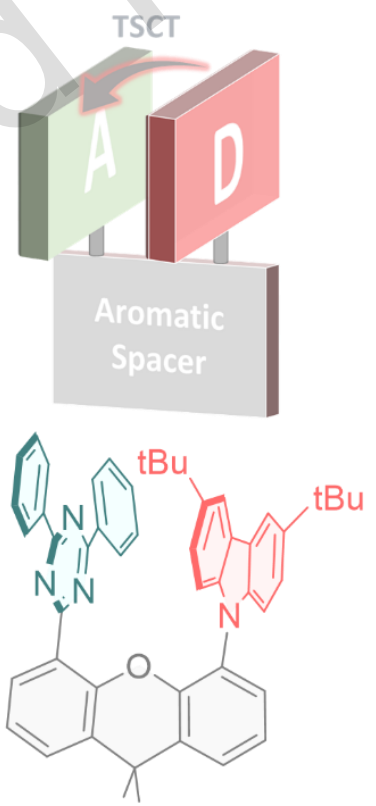

XtBuCT
3) Molecules involving the multiple resonnance effect<smiles>[R]Oc1ccccc1N(c1ccccc1O[R])c1ccccc1OC</smiles><smiles>c1ccc(N2c3ccccc3B3Nc4cccc2c4N(c2ccccc2)c2ccccc23)cc1</smiles>

DABNA-1

Adv. Mater. 2016, 2777

Figure 2: Molecular designs of TADF molecules and illustrative examples

Using one of these three strategies allows a spatial separation of the frontier molecular orbitals, the $\Delta \mathrm{E}_{\mathrm{ST}}$ being theoretically proportional to the overlap integral between the Highest Occupied 
Molecular Orbital (HOMO) and the Lowest Unoccupied Molecular Orbital (LUMO). While TADF molecules have allowed the development of very efficient OLEDs with varied colour emission, several key properties remain to be optimized for real-life applications in displays, such as the width of the emission spectrum to avoid a low color purity. ${ }^{[6]}$

In parallel to the development of OLEDs, optimization of this technology is still needed to face specific issues regarding their practical use. This is notably the case for numerous portable display devices, which use anti-glare filters including a linear polarizer and a quarterwave plate in the stack to limit the reflection of external light (Figure 3). Using this type of filters also implies the loss of up to $50 \%$ of the electroluminescence generated by the OLED. These challenges have therefore prompted investigations into a new generation of devices where the electroluminescence would be circularly polarized, ${ }^{[7]}$ and thus could pass these filters without any attenuation, leading to an increase in image brightness with lower power consumption.

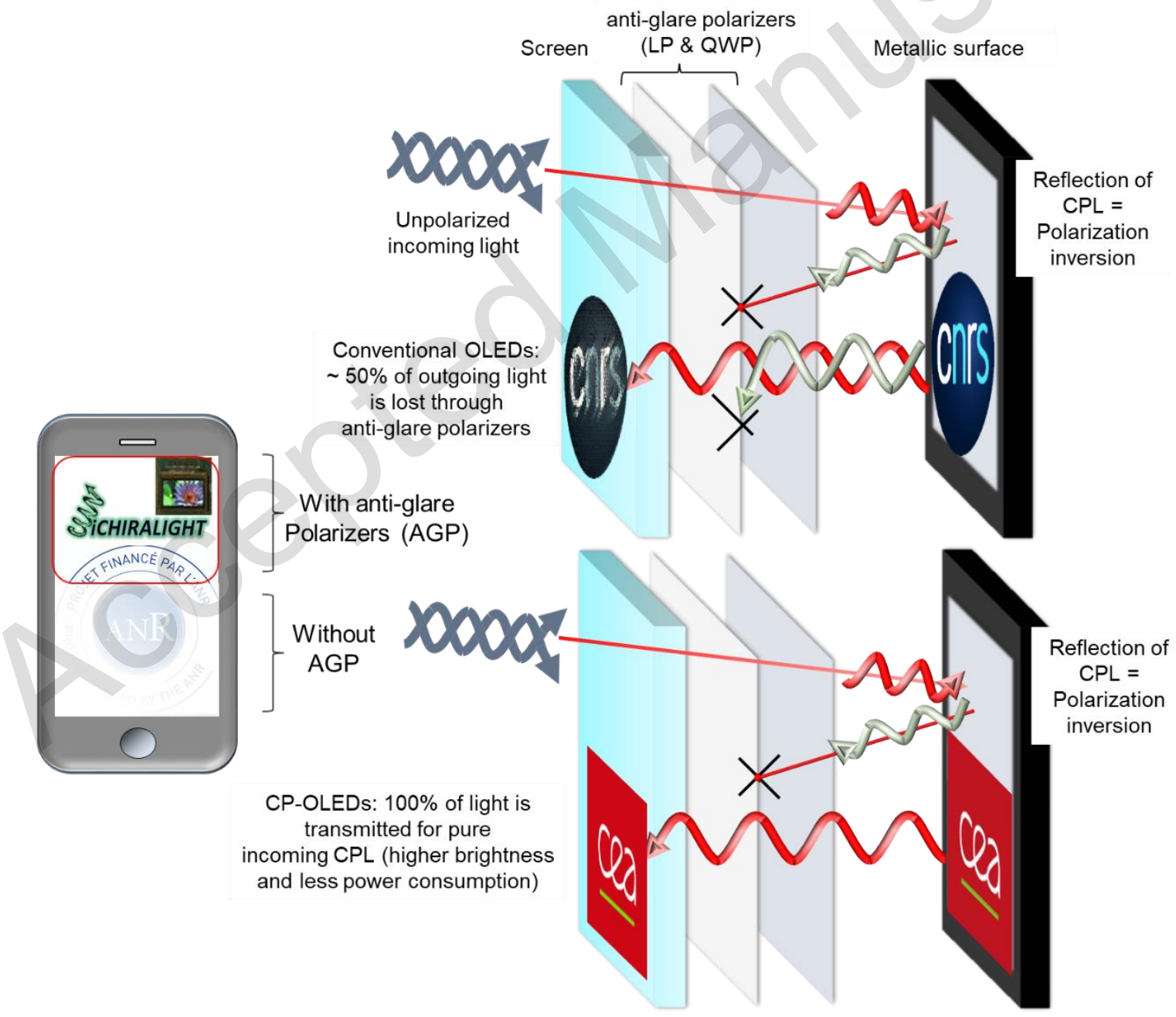

Figure 3: Illustration of the benefits of using CP-OLEDs for displays fitted with anti-glare filters including a linear polarizer (LP) associated to a quarter-wave plate (QWP). 
At the molecular level, it is well-known that the interaction of circularly polarized light with a chiral nonracemic compound gives rise to a specific response opposite in sign for the two enantiomers, which can be characterized using chiroptical spectroscopies. Among them, electronic circular dichroism (ECD) is by far the most popular and has been successfully applied to the determination of absolute configuration, enantiomeric excess of asymmetric reactions, molecular and supramolecular conformation analysis, and investigation of the supramolecular arrangement of chiral molecular and polymolecular aggregates. This spectroscopy refers to the differential absorption of left-handed $\left(A_{L}\right)$ and right-handed $\left(A_{R}\right)$ CP-light of a chiral molecule to probe its ground-state chiroptical properties. It is often quantified by the absorption dissymmetry factor, $g_{\text {abs }}$, defined as $\left(\mathrm{A}_{\mathrm{L}}-\mathrm{A}_{\mathrm{R}}\right) /\left(\mathrm{A}_{\mathrm{L}}+\mathrm{A}_{\mathrm{R}}\right)$, or, $\Delta \varepsilon / \varepsilon$, which is independent of the solution concentration (Figure 4 a).

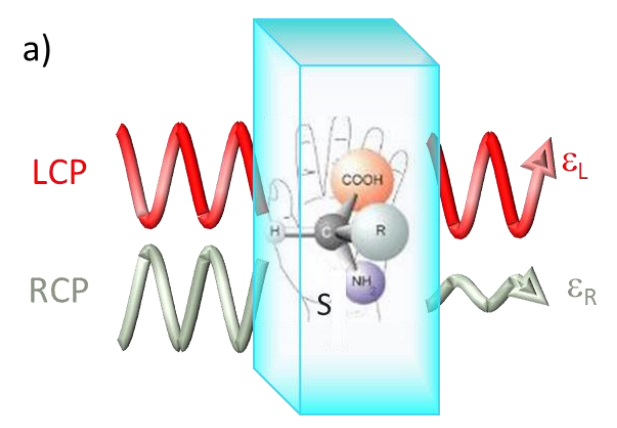

b)

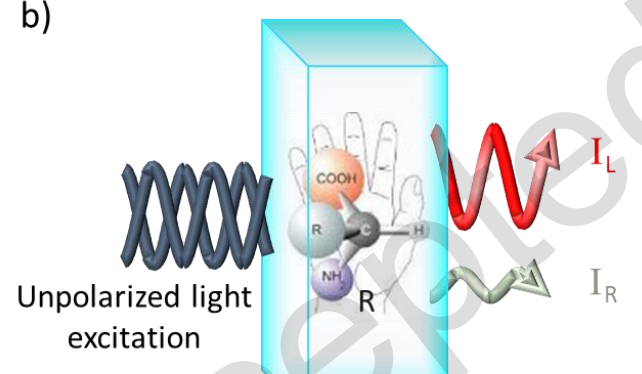

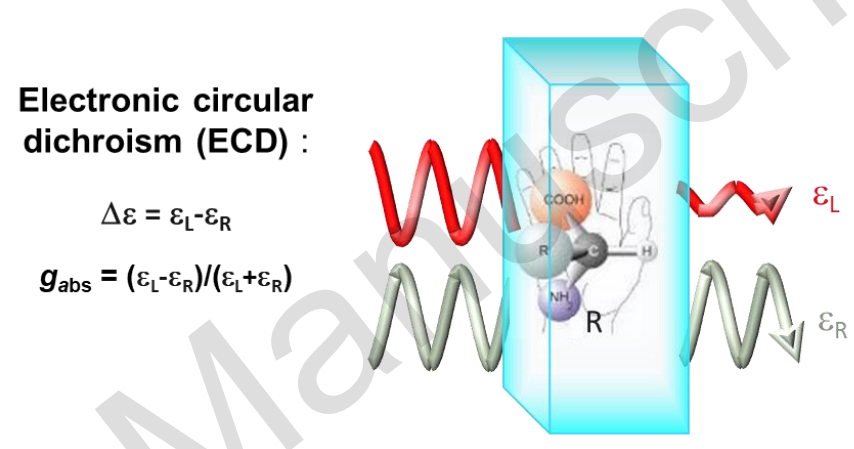

Circularly polarized luminescence $(\mathrm{CPL})$ :

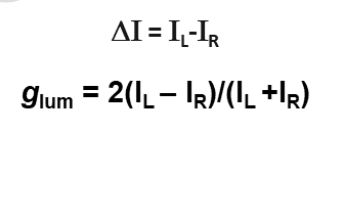

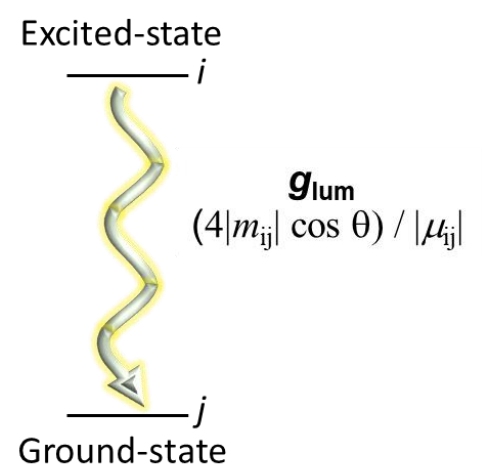

Figure 4: Illustration of the electronic circular dichroism (ECD) and circularly polarized luminescence (CPL) processes for a chiral luminescent molecule with the corresponding absorption and luminescence dissymmetry factors expressions. The latter is detailed with the involved electric and magnetic transition dipole moment vectors, $\mu$ and $m$, and $\theta$, the angle between them, for an electronic transition $\mathrm{i} \rightarrow \mathrm{j}$

For chiral luminescent molecules, a complementary process to ECD occurs in the radiative deactivation of their excited state, known as circularly polarized luminescence (CPL). Under unpolarized light or electrical excitation, a chiral luminophore is able to emit different 
intensities of right- or left-CP light, depending on the handedness of the enantiomer. The intensity of CPL emission is quantified in a similar way as $g_{\text {abs }}$ for the ECD but in emission intensity with a luminescence dissymmetry factor, $g_{\text {lum }}$, defined as $2\left(I_{\mathrm{L}}-I_{\mathrm{R}}\right) /\left(I_{\mathrm{L}}+I_{\mathrm{R}}\right)$, where $I_{\mathrm{L}}$ and $I_{\mathrm{R}}$ refer to the intensities of left- and right-circularly polarized emitted light, respectively. A total right- or left-circularly polarized luminescence is obtained when $g_{\text {lum }}$ values reach either 2 or +2 . As CPL spectroscopy is related to the chiroptical properties of the excited state of a chiral material, it is generally useful to associate the obtained $g_{\text {lum }}$ dissymmetry factor with the corresponding electronic transition $\left(\mathrm{i} \rightarrow \mathrm{j}\right.$ states) using the following equation: $g_{\text {lum }}=$ $4\left|m_{\mathrm{ij}}\right| \cdot\left|\mu_{\mathrm{ij}}\right| \_\cos \theta /\left(\left|m_{\mathrm{ij}}\right|^{2}+\left|\mu_{\mathrm{ijj}}\right|^{2}\right)$, which may be simplified to $g_{\text {lum }} \sim 4\left|m_{\mathrm{ij}}\right| /\left.\mu_{\mathrm{ij}}\right|^{2} \cos \theta$ for an electric dipole-allowed transition, where $m_{\mathrm{ij}}$ and $\mu_{\mathrm{ij}}$ are respectively the electric and magnetic transition dipole moments (for an $\mathrm{i} \rightarrow \mathrm{j}$ transition), and $\theta$, the angle between them. Accordingly, it becomes evident that $g_{\text {lum }}$ values will be maximized for chiral materials displaying magnetic dipoleallowed transitions and electric dipole-forbidden ones. As a result, and for a long time, lanthanide complexes have been the chiral molecular class of choice for the study of CPL phenomena due to their (formally Laporte forbidden) $\mathrm{f} \rightarrow \mathrm{f}$ transitions, which confer them large luminescence dissymmetry factor values $\left(\left|g_{\text {lum }}\right|>1\right) \cdot{ }^{[8]}$ In recent years however, (small) chiral organic molecules have been gaining momentum as potential emitters thanks to their often superior luminescence quantum yields, their tuneable photophysical and chiroptical properties, and their relatively simple integration in optoelectronic devices such as OLEDs, chiral photovoltaics and transistors. ${ }^{[9]}$ As a result, the main approach to develop CP-OLED devices relies on doping the device's emitting layer with a CPL emitter, which should ensure simultaneously high exciton conversion and a high degree of circular polarization. Up to now, the preparation of CPL emitters has been pursued using two different strategies. In the first one, the chiral element is embedded in the active fluorophore and therefore such compounds can be defined as intrinsically chiral CPL emitters ${ }^{[10]}$ (see the example of carbo[7]helicene, Figure 5, left). The second is based on the concept of chiral perturbation, where a chiral unit is placed in close proximity of the active fluorophore, thus conferring chiroptical properties to the latter (see Figure 5, right). 
Intrasically Chiral
Fluorophore
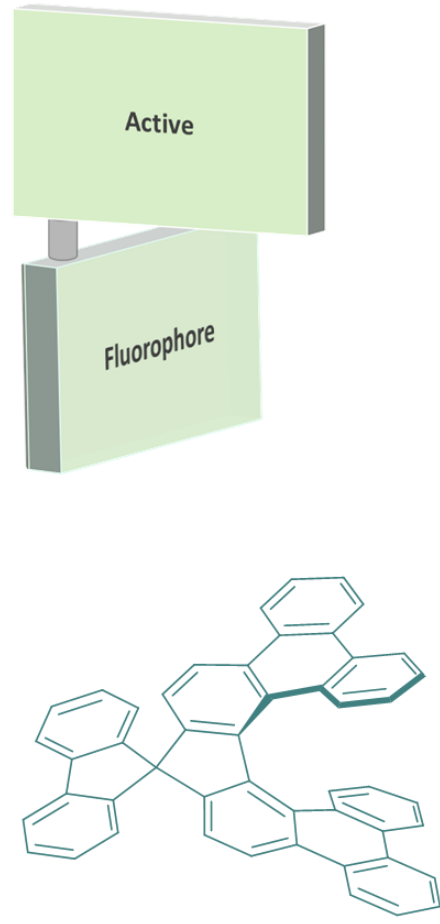

Carbo[7]helicene

J. Am. Chem. Soc. 2012, 4080
Chirally perturbated

Fluorophore

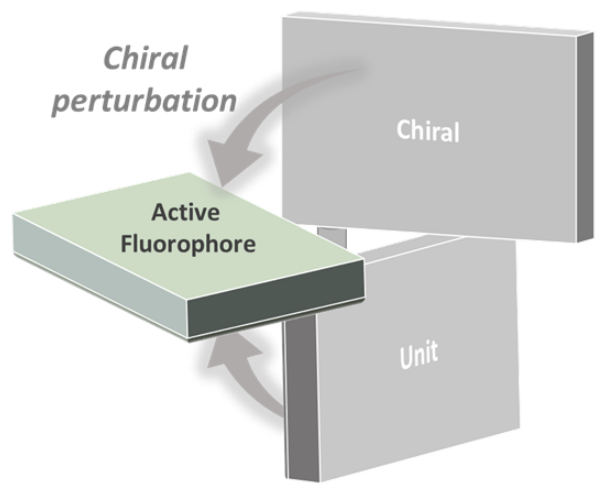

Chirally perturbated BODIPY

J. Am. Chem. Soc. 2014, 3346

Figure 5: Design strategies for CPL emitters

Whereas the use of intrinsically chiral CPL emitters allows reaching the highest values of $\left|g_{\text {lum }}\right|$ (currently up to $\left.10^{-2}\right),{ }^{[11]}$ the concept of chiral perturbation greatly facilitates synthetic access and performances screening, which are important considerations for applications in optoelectronics. Accordingly, current research involves the investigation of innovative CPL emitters to identify and understand the key electronic factors that govern the $g_{\text {lum }}$ at the molecular level.

In the context of novel OLED emitter development, molecules that efficiently combine TADF and CPL properties appear as an obvious research avenue. Indeed, they represent a valuable approach to ensure a high efficiency of both singlet and triplet excitons conversion for highperformance devices, as well as circularly polarized light emission to limit light attenuation arising from anti-glare filters. This progress report aims to describe the molecular designs merging TADF and CPL, discuss device performances, and finally highlight current and future challenges in this burgeoning field. ${ }^{[12]}$ 


\section{CP-TADF emitters: Molecular designs, photophysical and chiroptical properties}

Up to now, two main strategies have been applied to construct CP-TADF molecules, either based on inherently chiral TADF chromophores (using axial, planar or point chirality) or on the chiral perturbation of TADF units. While the former approach often gives higher $g_{\text {lum }}$ values, it requires resolution of enantiomers by different methods (chiral HPLC or resolution through diastereoisomeric separation). The latter strategy is in contrast based on syntheses starting with commercially available enantiopure products which greatly facilitates their large-scale synthesis and their application as emitters in OLEDs. Up to now, the vast majority of molecular designs utilize TICT molecules to generate the TADF properties, but recently some examples of TSCT chiral molecules have also been described. Here, the overview of these diverse molecular designs is organized in two main parts: 1) Design of intrinsically chiral CP-TADF emitters; 2) Design of CP-TADF emitters using the concept of chiral perturbation.

\subsection{Design of intrinsically Chiral CP-TADF emitters}

\section{1.a Intrinsically Chiral CP-TADF molecules involving point chirality}

One of the approaches used to prepare CPL emitters is the incorporation of a chiral carbon centre into the molecular structure. In their 2015 report, ${ }^{[13]}$ Hirata et al. described a fluorophore (DPHN) built around a triarylamine donor and naphthacene acceptor separated by a chiral carbon centre providing asymmetry and conjugation breaking between donor and acceptor units (Figure 6, top left). 


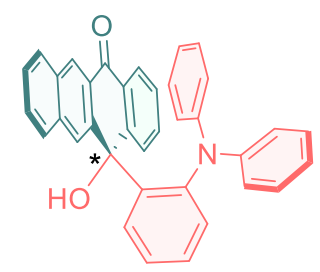

DPHN

$\left|g_{\text {lum }}\right|=1.1 \times 10^{-3}$

Jiang et al., J. Am. Chem. Soc. 2020

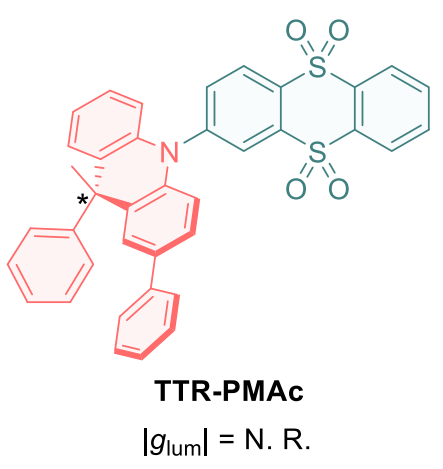

Yang et al., Mat. Horiz. 2021
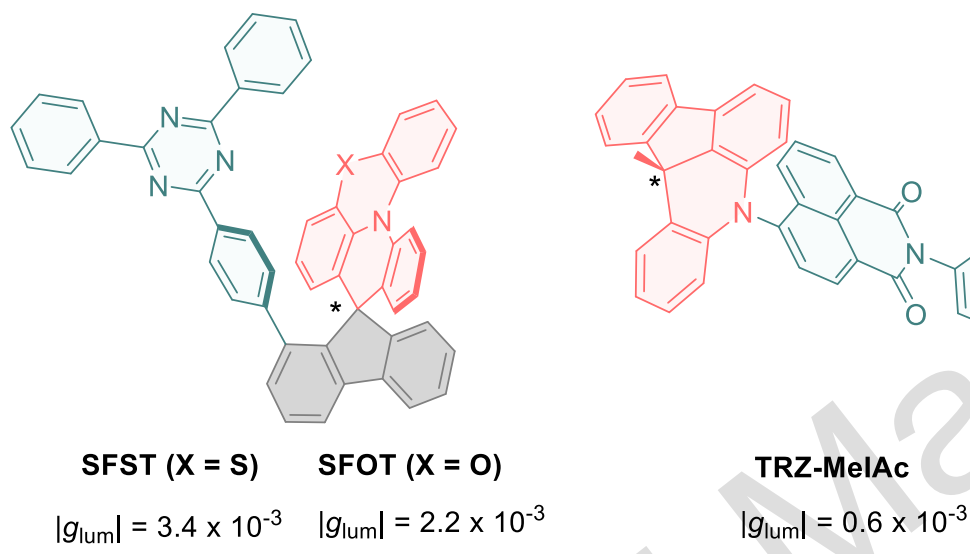

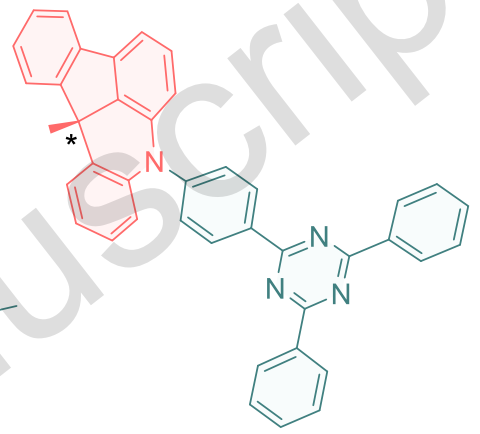

NID-MelAc

$\left|g_{\text {lum }}\right|=2.0 \times 10^{-3}$

Figure 6: Intrinsically chiral CP-TADF compounds involving point chirality

The calculated molecular orbitals of DPHN at $S_{1}$ showed spatial separation of the HOMO, located on the triarylamine unit, and the LUMO, located on the acene unit. In toluene, DPHN only exhibits prompt fluorescence with a PLQY of $4 \%$ and a fluorescence lifetime of $13.9 \mathrm{~ns}$. The experimental $\Delta \mathrm{E}_{\text {ST }}$ value of $0.19 \mathrm{eV}$ measured at low temperature suggests potential TADF properties. This molecule was used as a dopant at $9 \% \mathrm{w} / \mathrm{w}$ in an mCP matrix and exhibited a PLQY of $26 \%$ in doped thin-film state, a much higher value than the one measured in solution, and a long delayed fluorescent lifetime in the millisecond range. The chiral centre separating the donor and acceptor units leads to ECD activity, with a $\left|g_{\text {abs }}\right|$ of $1.2 \times 10^{-3}$, as well as CPL properties, with a $\left|g_{\text {lum }}\right|$ measured at $1.1 \times 10^{-3}$. Interestingly, a sign inversion between CPL and ECD spectra is observed and attributed to the significant conformational change occurring between $S_{0}$ and $S_{1}$. Although device applications have not been reported, this work describes the first example of CP-TADF molecules reported in the literature.

In 2020, Zhang et al. described TTR-PMac, ${ }^{[14]}$ a donor-acceptor compound where the chiral carbon centre is located on the donor unit (Figure 6, top right). Two geometries were predicted by theoretical modelling and observed through optical properties: one in a quasi-planar 
conformation between the donor and acceptor with a dihedral angle of $173^{\circ}$ and absorption at $\lambda_{\text {abs }}$ of $357 \mathrm{~nm}$; and one with a quasi-orthogonal conformation at an $86^{\circ}$ angle and a weaker absorption at $\lambda_{\text {abs }}$ of $400 \mathrm{~nm}$. A weak blue emission $(430 \mathrm{~nm})$ is observed for the former with an associated $\Delta \mathrm{E}_{\mathrm{ST}}$ of $0.38 \mathrm{eV}$, while the latter exhibits a stronger yellow emission $(577 \mathrm{~nm})$. In thin spin-coated films, the only observed emission is from the orthogonal form based on emission wavelength and intensity, and a $\Delta \mathrm{E}_{\mathrm{ST}}$ of $0.03 \mathrm{eV}$ was measured, indicating potential for TADF emission. This is supported by molecular orbital calculations that only show clear spatial separation of HOMO and LUMO on the orthogonal form, while the planar form exhibits significant overlap. Degassed fluorescence measurements, where emission intensity only increases for the yellow emission band attributed to the orthogonal conformer, confirmed that trend, and demonstrated increased fluorescence lifetimes in the absence of $\mathrm{O}_{2}$. ECD signal near the $\lambda_{\text {abs max }}$ regions attributed to each form is very weak, with a more intense signal in the donor's absorbance region, underlining poor chiral induction on the ICT band by the donor in the ground state. CPL spectra were recorded from spin-coated thin films on a CPL spectrometer and show very weak mirror-image signals for the $(R)$ - and $(S)$-enantiomers, precluding the determination

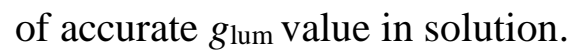

Jiang et al. have recently synthesised SFST and SFOT, ${ }^{[15]}$ where a triphenyltriazine acceptor is linked to either a chiral phentothiazine or phenoxazine, respectively, via a fluorene bridging unit (Figure 6, bottom left). In this design, the donor and acceptor groups are facing one another thanks to a chiral carbon centre on the aromatic spacer linking them. Because of restricted rotation, the D-A system is chiral as well. Based on X-ray diffraction, the donor and acceptor units were determined to be closer to parallelism in SFOT than SFST, with about $15^{\circ}$ dihedral angle difference between the two structures, conferring a more favourable arrangement for DA interaction to the former. For both compounds, absorption bands are observed at very similar wavelengths around 283 and $310 \mathrm{~nm}$, with a third band for SFOT at $347 \mathrm{~nm}$, in toluene. Emissions were observed at $512 \mathrm{~nm}$ for the two compounds in these conditions, with positive solvatochromism in both cases. $\Delta \mathrm{E}_{\mathrm{ST}}$ was determined experimentally at 0.052 and $0.053 \mathrm{eV}$ for SFST and SFOT, respectively, while theoretical calculations estimated these values at 0.026 and $0.012 \mathrm{eV}$. PLQYs were determined for both compound as a 30\% dopant in mCBP films, resulting in values of $44 \%$ for SFST and $77 \%$ for SFOT under air, and $53 \%$ and $89 \%$ under inert atmosphere, respectively. Prompt fluorescence lifetimes in these films were significantly different for $\operatorname{SFST}\left(\tau_{\mathrm{p}}=141 \mathrm{~ns}\right)$ and $\operatorname{SFOT}\left(\tau_{\mathrm{p}}=10 \mathrm{~ns}\right)$, while delayed fluorescence was of similar magnitude ( $\tau_{D}=6.78$ and $7.98 \mu \mathrm{s}$, respectively). These measurements underlined the $\mathrm{TADF}$ properties of the compounds that were expected from the $\Delta \mathrm{E}_{\mathrm{ST}}$ values. Mirror image 
ECD spectra were observed for both pairs of enantiomers, with very distinct spectral shape for each compound in toluene solutions. Corresponding $\left|g_{\text {lum }}\right|$ values of around $2.2 \times 10^{-3}$ and $3.4 \times$ $10^{-3}$ were observed for SFOT and SFST, respectively, in these conditions.

In a recent report, Yang et al. described another chiral donor linked to a variety of acceptors (Figure 6, bottom right). ${ }^{[16]}$ The chiral donor consists in an acridine derivative called MeIAc bearing a chiral carbon atom that has been described a few years earlier. ${ }^{[17]}$ This donor is coupled with two different acceptors: either a triazine derivative to obtain TRZ-MeIAc, or a naphthalimide derivative giving rise to NID-MeIAc.

Calculations on both compounds showed similar results: HOMOs are located on the chiral donor unit, whereas LUMOs are located on phenyl and triazine cycles for TRZ-MeIAc, and on the naphthalimide unit for NID-MeIAc.

Absorption spectra displayed two weak charge transfer bands at $367 \mathrm{~nm}$ for and $441 \mathrm{~nm}$ for TRZ-MeIAc and NID-MeIAc respectively. Emission maxima were centered at $476 \mathrm{~nm}$ for TRZ-MeIAc and $577 \mathrm{~nm}$ for NID-MeIAc in diluted toluene solution. Air-dependent PLQY of up to $49 \%$ for the blue emitter and $6 \%$ for the orange one were measured in solution. It is significant to note that these increase to $89 \%$ and $86 \%$, respectively, in mCPCN films under oxygen-free conditions. On top of that, authors have highlighted the TADF behaviour by measuring fluorescence lifetime under oxygen and oxygen-free conditions and observing both nanosecond and microsecond decays.

ECD measurements show mirror images for each pair of enantiomers. Furthermore, a weak but non-null signal is observed at the wavelength corresponding to the charge transfer band, showing that the chiral donor unit can induce chirality to the donor-acceptor system. $\left|g_{\text {lum }}\right|$ values of around $0.59 \times 10^{-3}$ and $2.0 \times 10^{-3}$ were measured for TRZ-MeIAc and NID-MeIAc, respectively. Interestingly, the authors have explained the difference in terms of $g_{\text {lum amplitude }}$ by theoretically determining the the electric $\left(\mu_{\mathrm{e}}\right)$ and magnetic $\left(\mu_{\mathrm{m}}\right)$ transition dipole moment, which indicate a strong influence of the ICT strength of the donor-acceptor combination on the chiroptical response of such compounds.

\section{1.b Intrinsically chiral CP-TADF molecules involving planar chirality}

The first example of a CP-TADF molecule with chiral [2.2]paracyclophane was reported by Zhao et al. in $2018 .^{[18]}$ In their paper, authors described two new molecules: $\boldsymbol{g}$-BNMe2-Cp and 
$\boldsymbol{m}$-BNMe2-Cp. These consisted of [2.2]paracyclophane where one ring carried a donor unit, a dimethyl amine, and the other bore the acceptor which is a dimesityl boron. The $g$ and $m$ correspond to the pseudo-gem (Figure 7, left) and the pseudo-meta positions of each donor substituent on the paracyclophane unit relative to the adjacent acceptor substituent.

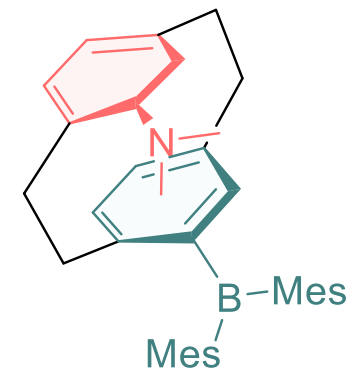

g-BNMe 2 -Cp

$\left|g_{\text {lum }}\right|=4.2 \times 10^{-3}$

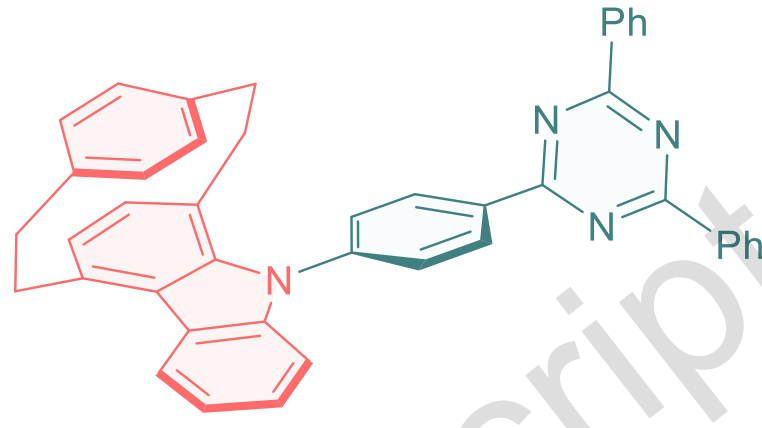

CzpPhTrz

$\left|g_{\text {lum }}\right|=1.2 \times 10^{-3}$

Figure 7: Cyclophane based CP-TADF molecules

Due to the spatial proximity of the central benzene rings, through-space charge transfer is enabled in these structures. DFT calculations showed a distribution of frontier orbitals as follows: the HOMO is mainly localized on the dimethyl amine unit and its parent benzene ring, whereas the LUMO is located on the dimesithyl boron and its corresponding benzene ring, for both molecules. This molecular design leads to a strong separation of frontier orbitals which is beneficial for TADF properties. $\boldsymbol{g}$-BNMe2-Cp exhibits a yellowish-green fluorescence in toluene $\left(\lambda \mathrm{em}_{\max }=531 \mathrm{~nm}\right)$, as well as good PLQY in degassed solution (46\%) which drops significantly in the presence of oxygen (around 7\%). Further experiments demonstrated TADF properties, with bi-exponential decays showing both short and delayed fluorescence, as well as a small $\Delta \mathrm{E}_{\mathrm{ST}}$ reaching $0.17 \mathrm{eV}$. Finally, chiroptical properties were solely investigated for $\boldsymbol{g}$ BNMe2-Cp due to its superior optical properties. Mirror-image spectra were observed in both ECD and CPL. Furthermore, $\left|g_{\text {lum }}\right|$ values were shown to reach $4.2 \times 10^{-3}$. This pair of enantiomers was not implemented in CP-OLED devices by the authors.

The second example of CP-TADF molecule based on [2.2]paracyclophane was been reported by Zysman-Colman et al. in 2019. ${ }^{[19]}$ This molecule, named CzpPhTrz (Figure 7, on the right), is based on a donor-acceptor system with a diphenyltriazine derivative as acceptor and a chiral 
carbazolophane ([2]paracyclo[2](1,4)carbazolophane) as donor. DFT calculations revealed a low frontier orbital overlap integral, with a LUMO located on the acceptor and the HOMO on the chiral carbazolophane unit. This molecule displayed a sky blue emission in toluene $\left(\lambda \mathrm{em}_{\max }\right.$ $=470 \mathrm{~nm}$ ) with a quantum yield of $70 \%$ in degassed solution. On top of that, experiments performed at low temperature (77 $\mathrm{K}$ in $10 \mathrm{wt} \%$ doped films in DPEPO matrix) allowed measuring a $\Delta \mathrm{E}_{\mathrm{ST}}$ of $0.16 \mathrm{eV}$. Temperature-dependent decays were also observed, confirming TADF properties. Chiroptical properties were investigated and both ECD and CPL display mirror image signals: $\left|g_{\text {abs }}\right|$ was measured at $6.0 \times 10^{-3}$ on the charge transfer band and $\left|g_{\text {lum }}\right|$ up to $1.2 \times 10^{-3}$ at maximum of luminescence. Finally, these pairs of enantiomers were implemented in OLED devices as dopants in a DPEPO matrix (10 wt\%). Electroluminescence was relatively similar to luminescence and led to an $\mathrm{EQE}_{\max }$ of $17 \%$. However, authors did not report the resulting polarization of the electroluminescence of the OLEDs.

\section{1.c Intrinsically chiral CP-TADF molecules involving axial chirality}

The last family of CP-TADF molecules based on intrinsically chiral molecules revolves around chirality borne by the acceptor unit. The first example of this category was published by Cheng et al. in 2019. ${ }^{[20]}$ In their report, they presented two molecules, yet only one displayed both CPL and TADF properties. This molecule, named BPO-PXZ, was based on a BINOL skeleton fused on their [2,3] and [2',3'] positions with chromones, themselves each substituted with a phenoxazine (see Figure 8, left). Due to the BINOL skeleton, a "cross" geometry is adopted between the two acceptor units, as well as an almost $90^{\circ}$ orientation between the donor and the acceptor. Thus DFT calculations locate the LUMO on the naphthyl acceptor unit whereas the HOMO is localized on the donors. Although BPO-PXZ is weakly fluorescent in solution, it possesses aggregation-induced emission enhancement (AIEE) behaviour, thus neat films and doped films (15 wt\% in TCTA) display good properties with yellow fluorescence in doped films $\left(\lambda \mathrm{em}_{\max }=568 \mathrm{~nm}\right)$ and PLQY up to $19 \%$. TADF properties were confirmed by the measurement of a small $\Delta \mathrm{E}_{\mathrm{ST}}$ of $0.059 \mathrm{eV}$, and a bi-exponential luminescence decay. Enantiomers of BPO-PXZ displayed mirror image ECD spectra, with a $\left|g_{\text {abs }}\right|$ of $4.7 \times 10^{-4}$, while $\left|g_{1 \text { lum }}\right|$ was estimated around $1.4 \times 10^{-3}$ in toluene solution. Unfortunately, the dissymmetry factors in thin film state were found to be lower, with values of $\left|g_{\text {lum }}\right|, 8.2 \times 10^{-4}$ in neat films and $7.7 \times 10^{-4}$ in doped films. 
Recently, in 2020, the Chen and Zheng groups have published a novel molecular design of CPTADF molecule. This approach is also based on the design of chiral dual emitters With two carbazoles as donor units the molecule described by Chen et al. is named $\mathbf{C z}-\mathbf{A x}-\mathbf{C N}$ (figure 8, middle left). ${ }^{[21]}$ Calculations show that the LUMO is located on the acceptor units bearing the cyano groups, and the HOMO is located on the carbazoles. The crystal structure highlights that the distance between a carbazole unit and the other furthest cyanobenzene moiety is short (2.66 $\AA$ ), thus authors evoke that TSCT can occur in this molecule. $\mathbf{C z}-\mathbf{A x}-\mathbf{C N}$ displays deep blue fluorescence in toluene $\left(\lambda \mathrm{em}_{\max }=458 \mathrm{~nm}\right)$ with moderate PLQY $(26 \%)$ and experiments performed in $\mathrm{THF} / \mathrm{H}_{2} \mathrm{O}$ mixtures uncovered the AIEE properties of this molecules. TADF properties were demonstrated using temperature-dependent experiments, showing biexponential decay, with the delayed component increasing with temperature. Chiroptical properties were investigated on both enantiomers, ECD (in film) and CPL (in solution and in films) of enantiomers exhibited mirror-image spectra. $\left|g_{\text {lum }}\right|$ was measured in toluene solution, reaching up to $5.5 \times 10^{-3}$, and was slightly lower in the film state $\left(4.7 \times 10^{-3}\right)$.

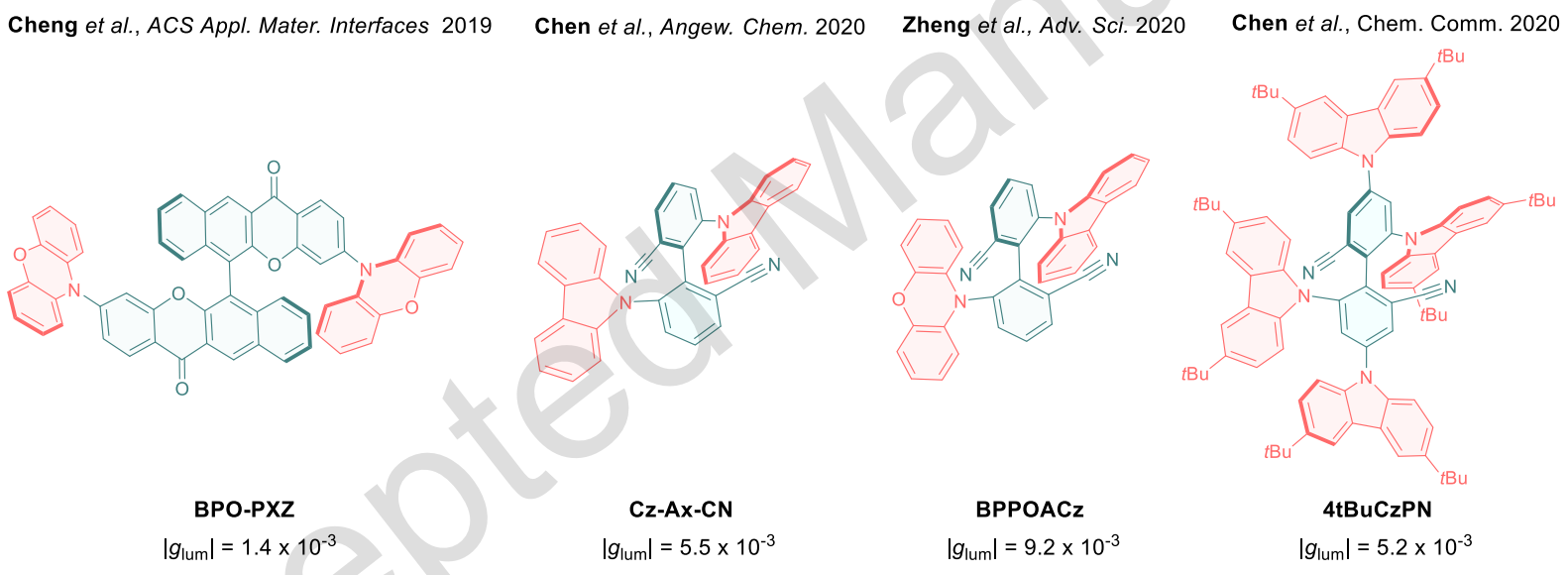

Figure 8: CP-TADF molecules involving chiral dual emitters

Zheng et al. have reported another molecule based on the same molecular design named BPPOACz. ${ }^{[22]}$ BPPOACz has the same acceptor core as $\mathbf{C z}-\mathbf{A x}-\mathbf{C N}$, but one carbazole and one phenoxazine as donors instead of two carbazoles (Figure 8, middle right). As expected, DFT calculations locate the LUMO on the central benzene rings while the HOMO is only found on the phenoxazine unit, without contribution from the carbazole moiety. Surprisingly, BPPOACz displays double emission fluorescence in toluene solution $\left(\lambda \mathrm{em}_{1}=384 \mathrm{~nm}, \lambda \mathrm{em} 2=\right.$ $543 \mathrm{~nm}$ ), which is attributed to two independent radiative transitions arising from both donorphenyl ring-acceptor systems. The latter is more intense and the former disappears when the 
product is dispersed as a dopant in a film of 2,6-DczPPy. This loss can be explained by a stronger intramolecular charge transfer promoted in the condensed state. PLQY was estimated at $86 \%$ in degassed toluene solution and TADF properties have been brought to light with the measurement of a small $\Delta \mathrm{E}_{S T}$ value $(0.04 \mathrm{eV})$, as well as a bi-exponential fluorescent decay with temperature-dependent behaviour. Finally, chiroptical properties were investigated in solution and in films. ECD and CPL spectra exhibited mirror images for enantiomers, and $\left|g_{\text {lum }}\right|$ values reached $9.2 \times 10^{-3}$ in toluene and $1.8 \times 10^{-2}$ in films. This example represents the highest $\left|g_{\text {lum }}\right|$ measured in toluene solution so far for CP-TADF molecules.

Finally, Chen et al. published another example based on the same molecular design. Compared to $\mathbf{C z - A x - P N , ~ t w o ~ a d d i t i o n a l ~ d o n o r ~ u n i t s ~ ( 3 , 6 - t e r t b u t y l c a r b a z o y l ~ i n ~ t h i s ~ c a s e ) ~ w e r e ~ i n c o r p o r a t e d ~}$ in positions 4 and $4^{\prime}$ of the dual acceptor, giving rise to $4 \mathbf{t B u C z P N}$ (Figure 8, right). ${ }^{[23]}$ 4tBuCzPN emits in the blue-green area $\left(\lambda \mathrm{em}_{\max }=497 \mathrm{~nm}\right)$ with a PLQY of $74 \%$ once incorporated in DPEPO films (25 wt $\%)$. TADF properties were evaluated through the measurement of $\Delta \mathrm{E}_{\mathrm{ST}}$ at low temperature $(0.05 \mathrm{eV}$ at $77 \mathrm{~K})$ and observation of delayed fluorescence lifetime at room temperature. Chiroptical properties were investigated and both ECD and CPL displayed mirror-image spectra, and dissymmetry factors in toluene solution and thin film state were comparable $\left(5.2 \times 10^{-3}\right.$ for both). To conclude, authors have implemented their molecules in OLED devices as dopants in a DPEPO matrix (25 wt\%). A high EQE of 20.8\% was obtained; yet no CPEL signal emanating from the OLED has been measured. Authors attributed the absence of signal to racemization of the product during the evaporation process. Indeed, although the Gibbs racemization free energy barrier is high $\left(37.2 \mathrm{kcal}^{-1} \cdot \mathrm{mol}^{-1}\right)$, the large size of the molecular structure accounts for a very high evaporation temperature.

Since the first example of CP-TADF molecule reported in late 2015, numerous designs of intrinsically chiral CP-TADF emitters have been explored, mostly based on electron donor moieties bearing a chiral center, paracyclophane electron donor derivatives, or axial chirality of dual emitters. Most of these examples involve a TICT framework, except for SFST/SFOT and $\boldsymbol{g}$-BNMe2-Cp which display interesting chiroptical properties. In terms of emission colours, most of these molecular designs allow the synthesis of blue and green CP-TADF emitters, whereas orange/red fluorophores are less described due to the inherent difficulties in designing high-performance red-emitting TADF molecules. Concerning the overall performances (PLQY and $g_{\text {lum }}$ ), the recent design described by Chen and Zheng involving intrinsically chiral dual 
emitters seems to be the most promising even if the origin of the strong chiroptical activities of these molecules remains to be clarified.

Such promising results clearly highlight the need of further molecular engineering and structure/properties relationships to identify the key parameters governing the combination of CPL and TADF properties.

\subsection{Design and application CP-TADF emitters using the concept of chiral perturbation}

\section{2.a. BINOL based CP-TADF molecules}

One year after Hirata et al.'s early report of the first CP-TADF molecule, some of us described a new chiral TADF chromophore based on chiral perturbation, hereinafter named $\mathbf{A 1}{ }^{\text {[24] }}$ (Figure 9, top left). This CP-TADF molecule is composed of an active fluorophore unit consisting of terephthalonitrile as the acceptor and two carbazoyl units as the donors, attached to an enantiopure chiral BINOL. DFT calculations displayed a remarkable separation of frontier orbitals with the HOMO mainly on the carbazole units and the LUMO on the terephthalonitrile, which could imply a small $\Delta \mathrm{E}_{S \mathrm{ST}}$. It is furthermore important to notice that no frontier orbital coefficients are located on the chiral unit. Molecule A1 displayed green fluorescent emission in toluene $\left(\lambda \mathrm{em}_{\max }=517 \mathrm{~nm}\right)$ with PLQY up to $28 \%$ in aerated solution. Once purged with argon gas, this value increased to $53 \%$ and a bi-exponential emission decay was obtained, ascertaining TADF properties. As detailed above, although the chiral part is not directly involved in the frontier orbitals, A1 displayed ECD and CPL activities, corroborating an effective chiral perturbation process. An interesting change of sign between ECD and CPL measurement can be observed, suggesting a modification of geometry between the fundamental state and $\mathrm{S}_{1}$. 

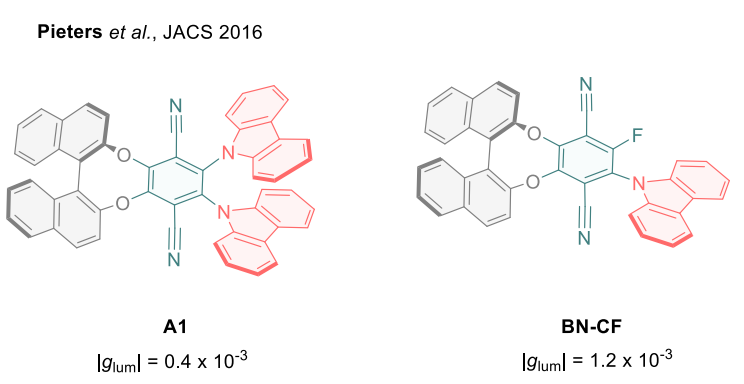

Tang et al., Adv. Funct. Mat. 2018
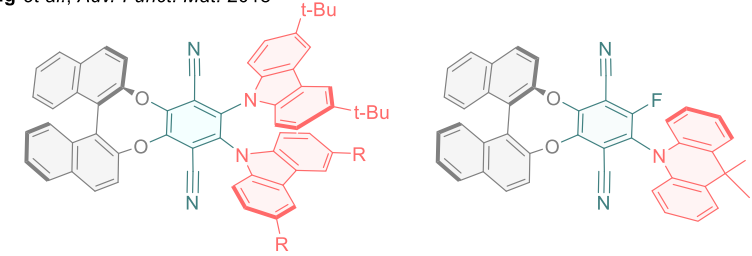

$B N-C C B(R=H) \& B N-D C B(R=t-B u)$

$\left|g_{\text {lum }}\right|=1.2 \times 10^{-3} \quad\left|g_{\text {lum }}\right|=0.8 \times 10^{-3}$

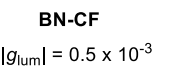

Zheng et al., Adv. Mat. 2019

Zheng et al., J. Chem. Mater C 2019

Huang et al., J. Chem. Mater C 2019
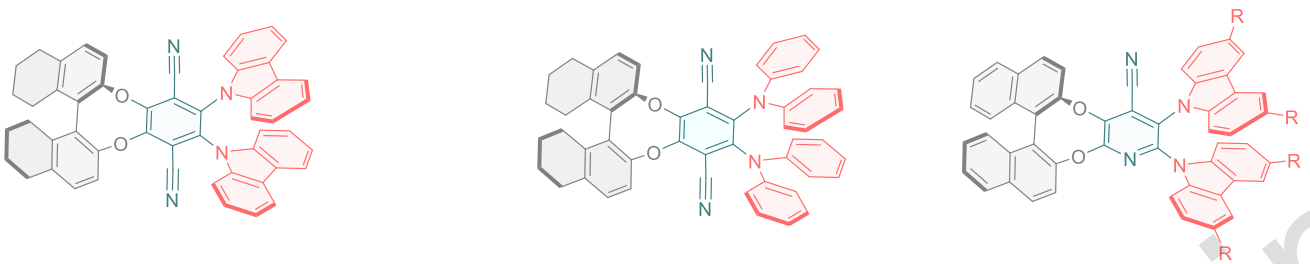

OBN-Cz

OBN-DPA

$\left|g_{\text {lum }}\right|=1.8 \times 10^{-3}$

CPDCz $(R=H) \&$ CPDCB $(R=t-B u)$

$\left|g_{\text {lum }}\right|=0.35 \times 10^{-3} \quad\left|g_{\text {lum }}\right|=0.5 \times 10^{-3}$

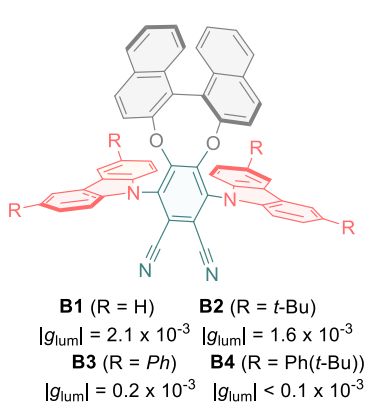

Pieters et al., Adv. Funct. Mat. 2020

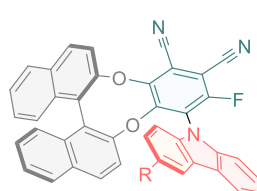

$\mathrm{C}^{\prime} 1(\mathrm{R}=\mathrm{H}) \quad \mathrm{C}^{\prime} \mathbf{2}(\mathrm{R}=t-\mathrm{Bu}) \quad \mathrm{C}^{\prime} \mathbf{3}(\mathrm{R}=\mathrm{Ph})$

$\left|g_{\text {lum }}\right|=1.1 \times 10^{-3} \quad\left|g_{\text {lum }}\right|=1.0 \times 10^{-3} \quad\left|g_{\text {lum }}\right|=1.1 \times 10^{-3}$

C1 $(\mathrm{R}=\mathrm{H}) \quad \mathrm{C2}(\mathrm{R}=\mathrm{t}-\mathrm{Bu}) \quad \mathrm{C3}(\mathrm{R}=\mathrm{Ph})$ $\left|g_{\text {lum }}\right|<0.1 \times 10^{-3} \quad\left|g_{\text {lum }}\right|=0.7 \times 10^{-3}$

Figure 9: CP-TADF emitters using the chiral perturbation strategy

On a bespoke CPL spectrometer, $\left|g_{\text {lum }}\right|$ at the maximum of emission reached $1.3 \times 10^{-3}$, however more recent experiments with a commercially available CPL-spectrometer showed a more moderate value of $0.4 \times 10^{-3}$. Finally this pair of enantiomers was implemented in OLED devices as dopants in an mCP matrix (20 wt \%). This application resulted in electroluminescence spectra comparable to the luminescence ones, and a moderate EQE of 9.1\%. Albeit no racemization has been detected during the fabrication process of the OLEDs, we were not able to measure $\mathrm{CPL}$ emanating from these devices using a quarter wave plate/linear polarizer apparatus probably because of the small dissymmetry factor and the architecture chosen at that time to build the OLED (see part 4.2 for more detailed discussion).

Based on the same molecular design, Tang et al. reported in 2018 a set of 4 other molecules (Figure 9, top middle and top right). ${ }^{[25]}$ Whereas two identical carbazole units were present in A1, the newer members of this family were composed of either only one donor unit (a carbazole 
for BN-CF or a dimethylacridine for BN-AF), leaving a fluorine atom on the central acceptor unit, or two donors (two 3,6-ditertbutylcarbazoles for BN-DCB, or one 3,6-ditertbutylcarbazole and one carbazole for BN-CCB). Frontier orbitals showed the same distribution as the initial example, that is, the HOMO on the donor unit(s) and the LUMO on the acceptor unit. TD-DFT estimated $\Delta \mathrm{E}_{\text {ST }}$ values ranging from $0.02 \mathrm{eV}$ for $\mathbf{B N}-\mathbf{D C B}$ to $0.15 \mathrm{eV}$ for $\mathbf{B N}-\mathbf{C F}$, which are compatible with TADF emission. Photophysical characterization led to fluorescence spectra from greenish-blue to yellowish orange depending on the compound ( $\lambda \mathrm{em}_{\max }=495$ to $585 \mathrm{~nm}$ in toluene). Most data collected were based on neat films or doped films prepared with each compound. In neat films, PLQY of BN-CF, BN-AF, BN-DCB and BN-CCB reach 53\%, 38\%, $22 \%$ and 20\%, respectively, and characteristic bi-exponential decays were observed, confirming the TADF properties. Furthermore, AIEE properties of every compound were also studied, where it appeared by gradually increasing the proportion of water in an organic solvent (in this case THF), authors observed first a decrease of the luminescence intensity (up to 60\% of water in THF) followed by an upsurge of fluorescence at a higher proportion of water (by a factor reaching almost 12 in the case of $\mathbf{B N}-\mathbf{A F}$ ). In the same way as the parent example for this family, these 4 new molecules also exhibited chiroptical activity. ECD spectra present a $\left|g_{\text {abs }}\right|$ ranging from $\sim 2.5 \times 10^{-4}$ for $\mathbf{B N}-\mathbf{A F}$ to $7.4 \times 10^{-4}$ for $\mathbf{B N}-\mathbf{C F}$ in toluene solution, while CPL measurement led to $\left|g_{\text {lum }}\right|$ reaching $5.0 \times 10^{-4}$ for $\mathbf{B N}-\mathbf{A F}, \sim 8.0 \times 10^{-4}$ for $\mathbf{B N}-\mathbf{D C B}$ and 1.2 $\times 10^{-3}$ for BN-CCB and BN-CF. Surprisingly, both $g_{\text {abs }}$ and $g_{\text {lum }}$ values were exacerbated in neat films (by a factor between 2 and 7 for $g_{\text {abs }}$ and between 30 and 40 for $g_{\text {lum }}$ ). According to the authors, this chiroptical amplification may be attributed to the formation of chiral aggregates in the neat thin-film state.

In 2019, Huang et al. published other examples of CP-TADF molecules based on the same molecular design with the use of 4-cyanopyridine as the acceptor unit. ${ }^{[26]}$ Two new molecules were presented, namely CPDCz and CPDCB (Figure 9, middle right). The use of a cyanopyridine derivative did not change the HOMO-LUMO distribution, remaining on donor and acceptor, respectively, thus those orbitals did not involve the BINOL unit either. These pairs of enantiomers displayed fluorescence centered on 510 and $520 \mathrm{~nm}$ with PLQY in THF solution reaching 27\% and 37\% (for CPDCz and CPDCB respectively). Luminescence measurement at low temperature and transient luminescence decays were in accordance with a TADF behaviour. Surprisingly, in solution, compound CPDCz does not exhibit an ECD signal on the first absorption transition, whereas its tertbutyl analog showed a weak signal on the same position. On the other hand, neat films of both compounds displayed non-null ECD signals on 
every absorption area. CPL measurement led to low $\left|g_{\text {lum }}\right|$ values in solution (around $3.2 \times 10^{-4}$ and $2.7 \times 10^{-4}$ for $\mathbf{C P D C z}$ and $\mathbf{C P D C B}$ respectively). Surprisingly, measuring $g_{\mathrm{pl}}$ in neat films processed by spin-coating gave slightly higher values (around $3.5 \times 10^{-4}$ and $4.9 \times 10^{-4}$ for CPDCz and CPDCB respectively), and authors suggest that this increase could come from the slight orientation of chiral aggregates during the fabrication of films.

The same year, Zheng et al. reported two very similar new examples of CP-TADF molecules. While Tang et al. modified the donor unit(s) and Huang et al. modified the acceptor unit, in these reports Zheng et al. modified on the one hand the chiral unit, and then, on the other hand the donor units. Indeed, instead of using BINOL, the hydrogenated derivative octahydrobinaphthol (shortened to o-BINOL) was used, with carbazole donors for their first report, OBN$\mathbf{C z},{ }^{[27]}$ or diphyenylamine donors in $\mathbf{O B N}^{-D_{P A}}{ }^{[28]}$ (Figure 9, middle left and middle center). Similarly to the previous examples, DFT calculations present a HOMO-LUMO localization on donor-acceptor units, without any distribution on the o-BINOL unit. Photophysical measurements showed green fluorescence in toluene, with $\lambda \mathrm{em}_{\max }=503 \mathrm{~nm}$ for $\mathbf{O B N}-\mathbf{C z}$ and $\lambda \mathrm{em}_{\max }=538 \mathrm{~nm}$ for OBN-DPA. For OBN-Cz, the PLQY increased from 18\% to $58 \%$ after purging the solution with nitrogen, and both compounds displayed bi-exponential decays, confirming TADF properties. On top of that, authors have demonstrated that $\mathbf{O B N}-\mathbf{C z}$ and OBN-DPA exhibit AIEE properties. Circular dichroism spectra displayed mirror image signals of both enantiomers for every compound and a different CPL activity depending on the product in toluene solution, reaching $\left|g_{\operatorname{lum}}\right|$ values of $5.1 \times 10^{-4}$ for $\mathbf{O B N}-\mathbf{C z}$ and $1.8 \times 10^{-3}$ for $\mathbf{O B N}$ DPA. Surprisingly, $\left|g_{\text {lum }}\right|$ values were found to be slightly higher in neat film $\left(2.1 \times 10^{-3}\right)$ or codoped film $\left(1.8 \times 10^{-3}\right)$ for $\mathbf{O B N}-\mathbf{C z}$ in contrast with the huge chiral amplification measured for the BN-(AF/DCB/CF/CCB) series despite structural similarities. This slight increase of dissymmetry factor, moving from solution to thin film state was also observed for OBN-DPA but the multiplication factor was lower ( $\left|g_{\text {lum }}\right|$ reached $2.2 \times 10^{-3}$ in neat film).

Other examples of CP-TADF molecules based on chiral perturbation with a BINOL unit were recently published by our group. ${ }^{[29]}$ In this study, we investigated the influence of isomerism in such BINOL-based TADF molecules with the aim to decipher the key structural and electronic parameters allowing to maximize chiral perturbation while preserving TADF properties. To this end, three series of molecules, B, C and C', were synthesized and studied (Figure 9, bottom). Molecules $\mathbf{B}$ are based on a phthalonitrile moiety as the acceptor unit, with BINOL linked on the 4 and 5 positions, and different carbazole derivatives on positions 3 and 6 acting as electron 
donors. On the other hand, compounds $\mathbf{C}$ and $\mathbf{C}^{\prime}$ were obtained starting from phthalonitrile, the BINOL unit being in positions 3 and 4 . In the case of $\mathbf{C}^{\prime}$ only one carbazole derivative was introduced as the donor in position 5, whereas two donors were connected in positions 5 and 6 for compounds C. For every compound, DFT calculations presented a similar orbital distribution of HOMO and LUMO mainly located on donor(s) and acceptor units, respectively, whereas no frontier orbital coefficients were located on the BINOL unit in any of these cases. All of those compounds are fluorescent with colors ranging from blue $\left(\mathbf{B 1}, \lambda \mathrm{em}_{\max }=469 \mathrm{~nm}\right)$ to green $\left(\mathbf{C 3}, \lambda \mathrm{em}_{\max }=519 \mathrm{~nm}\right)$, low to moderate QY in toluene (from $2 \%$ to $30 \%$ in aerated solutions) and AIEE behaviour. Bi-exponential luminescent decays were observed in PMMA matrix confirming the TADF properties for all of those molecules. ECD spectra presented nonnull signals at the first absorption band for every compound except $\mathbf{C 1}$ and $\mathbf{C 2}$, implying that chiral perturbation is effective at the fundamental state for all others molecules. DFT calculations have been performed for each molecule to study the influence of isomerism and carbazole substitution on the efficiency of the chiral perturbation at the ground state (by evaluating the electric and magnetic transition dipole moments of the $S_{0}$ to $S_{1}$ transition). The results point out the favourable orientation of these moments for $\mathbf{B}$ and the relatively high value of $\mu_{\mathrm{m}}$ for compounds $\mathbf{C}^{\prime}$ and, as expected, a great influence of the carbazole substitution on theses crucial parameters for the chiroptical properties. It is worth noticing that compound B1 displayed the highest $\left|g_{\text {lum }}\right|$ value reported so far in solution for CP-TADF molecules based on chiral perturbation $\left(2.1 \times 10^{-3}\right.$ at $\left.\lambda \mathrm{em}_{\max }\right)$ and that no significant chiroptical properties amplification were measured switching from solution to thin film states (fabricated by spin coating or thermal deposition).

\section{2.b. 1,2-diaminocyclohexane based CP-TADF molecules}

In 2018, Chen et al. published their first example of a CP-TADF molecule named CAl-Cz (Figure 10, left). ${ }^{[30]}$ Based on a TADF active scaffold previously reported by their group, ${ }^{[31]}$ this new molecular design consisted in the use of an enantiopure diaminocyclohexane substituted by two phthalimide acceptor units. On each of these, two carbazole units used as donors were covalently linked onto positions 4 and 5 of the benzene ring. 


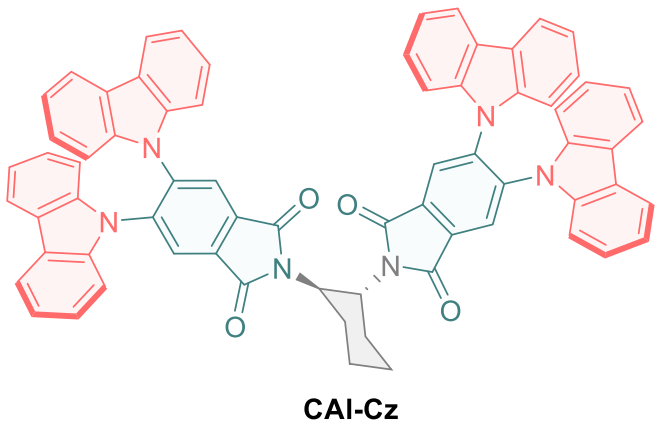

$\left|g_{\text {lum }}\right|=1.1 \times 10^{-3}$

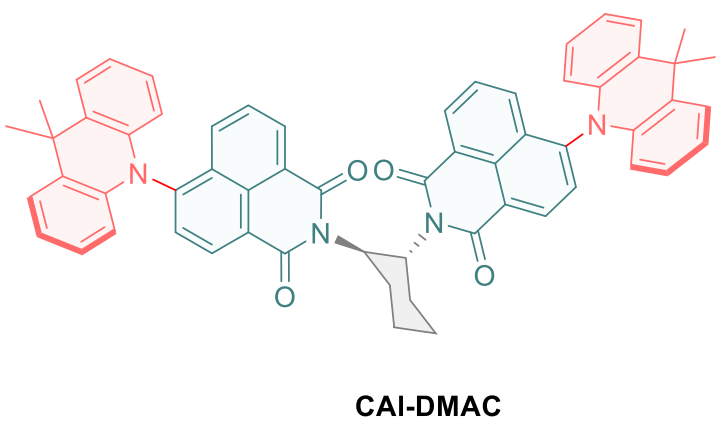

$\left|g_{\text {lum }}\right|=0.9 \times 10^{-3}$

Figure 10: CP-TADF molecules based on the trans-1,2-diaminocyclohexane scaffold

DFT calculations allowed to visualize the HOMO mainly localized on carbazole units while the LUMO was located on the phthalimides. This example can be classified in chiral perturbation because the chiral unit does not directly contribute to the frontier orbitals (for other chiral fluorophores based on the same 1,2-diaminocyclohexane scaffold the chiroptical properties were shown to be originating from chiral excitonic coupling between the two active fluorophores). ${ }^{[32]} \mathbf{C A l}-\mathbf{C z}$ is fluorescent in the green area $\left(\lambda \mathrm{em}_{\max }=515 \mathrm{~nm}\right)$ with a moderate PLQY of $18 \%$ in toluene solution. However, the latter increased to $41 \%$ in neat film and up to $80 \%$ in doped films (15\% in mCBP matrix). On top of that, experiments displayed time- and temperature-dependent bi-exponential decays confirming the TADF behaviour of this pair of enantiomers. Almost mirror image ECD and CPL spectra of the enantiomers were obtained, characterized by a non-null ECD signal in the region of the first electronic transition absorption, and a $\left|g_{\text {lum }}\right|$ of $1.1 \times 10^{-3}$ in toluene solution at $\lambda \mathrm{em}_{\max }$.

Later, the same group used the 1,2-diaminocyclohexane scaffold to synthesize an orange CPTADF emitter. In this example, the acceptors were swapped for 1,8-naphthalimides which were then substituted in position 3 by a dimethylacridine donor, giving rise to CAI-DMAC (Figure 10, right). ${ }^{[33]}$ The distribution of frontier orbitals remained similar to the previous example, i.e. HOMO on the donors (dimethylacridine) and LUMO on the acceptors (naphthalimide). Once again, the diaminocyclohexane unit showed no contribution to either of the frontier orbitals. CAI-DMAC presents yellow-orange fluorescence ( $\lambda \mathrm{em}_{\max }=583 \mathrm{~nm}$ in toluene) with PLQY up to $26 \%$ in film under aerated condition. Bi-exponential decays and oxygen-dependent PLQY were measured proving the TADF character of this compound. ECD and CPL spectra were each mirror-imaged, and a $\left|g_{\text {lum }}\right|$ value comparable to that of CAl-Cz, reaching $9.2 \times 10^{-4}$ was 
determined in toluene solution. Finally, the emitter was used as a dopant (6 wt \% in CBP matrix) in OLED devices displaying a good EQE of up to $12 \%$. Nevertheless, the chiroptical properties of these OLEDs were not reported.

Using chiral perturbation to design CPL active molecules has the advantage of facilitating synthetic access compared to intrinsically chiral ones, thanks to commercially available chiral scaffolds arising from decades of research in asymmetric catalysis. In this context, the entirety of examples reported so far involves TICT emitters and the design of orange/red light emitting compounds remains scarce ( 2 examples out of 21). A better understanding of the key parameters, such as the proximity and relative orientation of the chiral unit with respect to the active TADF unit, or the influence of the nature of the donor and acceptor moiety, could allow maximizing the efficiency of the chiral perturbation. The latter remains to be optimized in order to reach the state-of-the-art dissymmetry factors of intrinsically chiral CP-TADF molecules. While up to now only 2 types of perturbating chiral units have been studied (BINOL derivatives and the diaminocyclohexane scaffold), the use of other moieties coming from other facets of chemistry (organic and organometallic chemistry, natural products,...) could also be a way to increase the dissymmetry factors and unlock the technological potential and commercial application of CPL emitters in a broad sense.

\section{CP-TADF OLEDs}

Among all the CP-TADF emitters described above, the performances of some of them have also been evaluated either as dopants or directly as neat films in CP-OLEDs. Stack compositions (including the nature and thickness of the cathode), electroluminescence, and chiroptical performances of all CP-TADF OLEDs described so far are depicted in Figure 12 and gathered in Table 1. Owing to the varied device architectures, a direct comparison of the obtained performances in terms of efficiency and luminance is rather difficult and appears not strongly relevant for this progress report. As for their achiral TADF counterparts in conventional unpolarized OLEDs, the characterization of CP-OLED performances involves the external quantum efficiency defined earlier, as well as brightness and roll-off efficiency. Briefly, we would like to mention that the studied CP-OLEDs based on chiral TADF emitters exhibit promising to high-end external quantum efficiencies, up to $32 \%$, due to notably both a high luminescence quantum yield and efficient TADF properties. While these results are obviously important for OLED applications, it seems more interesting in our opinion to focus on the 
obtained CP electroluminescence and the detection methods to characterize it, since this represents the added value of the CP-OLED technology that truly distinguishes them from other device families.

Similarly to CPL measured in solution, the circularly polarized electroluminescence (CPEL) dissymmetry factor, $g_{\mathrm{El}}$, is defined as twice the ratio between the intensity difference of leftand right-CPEL, over the total generated electroluminescence, $2\left(E l_{\mathrm{L}}-E l_{\mathrm{R}}\right) /\left(E l_{\mathrm{L}}+E l_{\mathrm{R}}\right)$. Detection and characterization of electroluminescence polarization rely on the same concept than the one used for CPL spectroscopy, namely the conversion of the emitted right- and left-circular polarized light into a linearly polarized beam, which is then detected at a photomultiplier (Figure 11). Therefore, such measurements can be readily performed by placing a quarter-wave plate and a linear polarizer between the CP-OLED and the detector. In this configuration, rightand left-circular polarized light can be selectively detected by adapting the fast and slow axis of the quarter-wave plate together with the one of the linear polarizer.

The other method consists in directly placing the CP-OLED in the sample holder of a CPL spectrometer, and taking advantage of the photoelastic modulator (PEM), which alternatively converts right- and left-circular polarized electroluminescence to linearly polarized light. Accordingly, one can characterize the circular polarization of electroluminescence $\left(g_{\mathrm{El}}\right)$ and compare it to the intensity of CPL observed for the chiral emitter, either in solution $\left(g_{\text {lum }}\right)$ or in thin-film state film $\left(g_{\mathrm{pl}}\right)$. These three parameters are also indicated in Table 1 for the sake of comparison of the obtained CP-OLED performances (when those 3 values have been reported). 


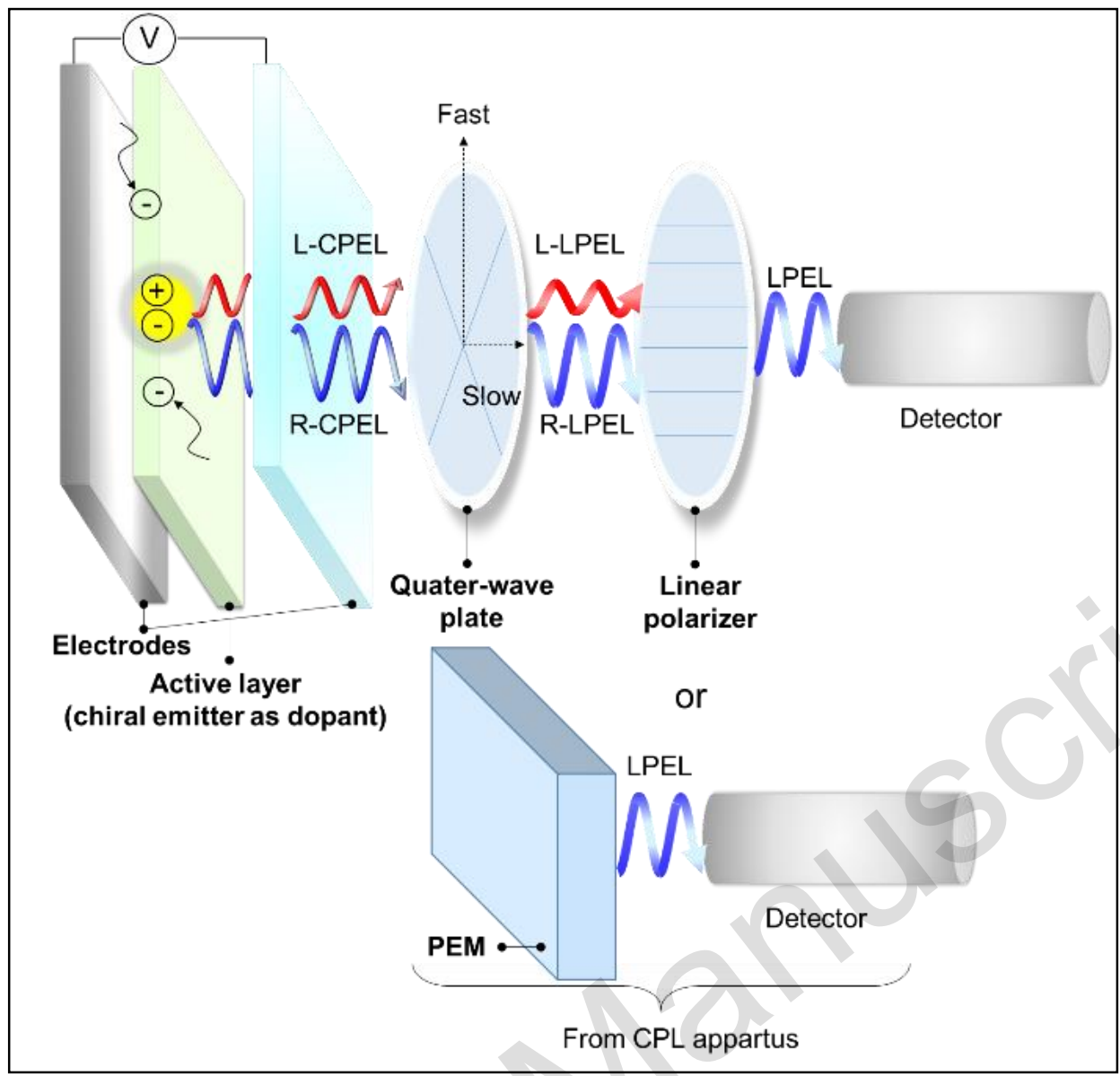

Figure 11: Schematic illustration of the two detection methods used to characterize the circularly polarized electroluminescence of CP-OLEDs involving either the combination of a quarter-wave plate and a linear polarizer (top) or the use of a CPL spectrometer based on a photoelastic modulator (PEM, bottom). 
Bottom-Emission CP-OLED

CP-TADF Emitter
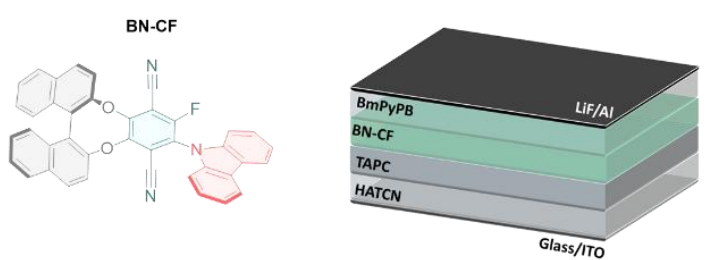

CP-TADF Emitter OBN-CZ
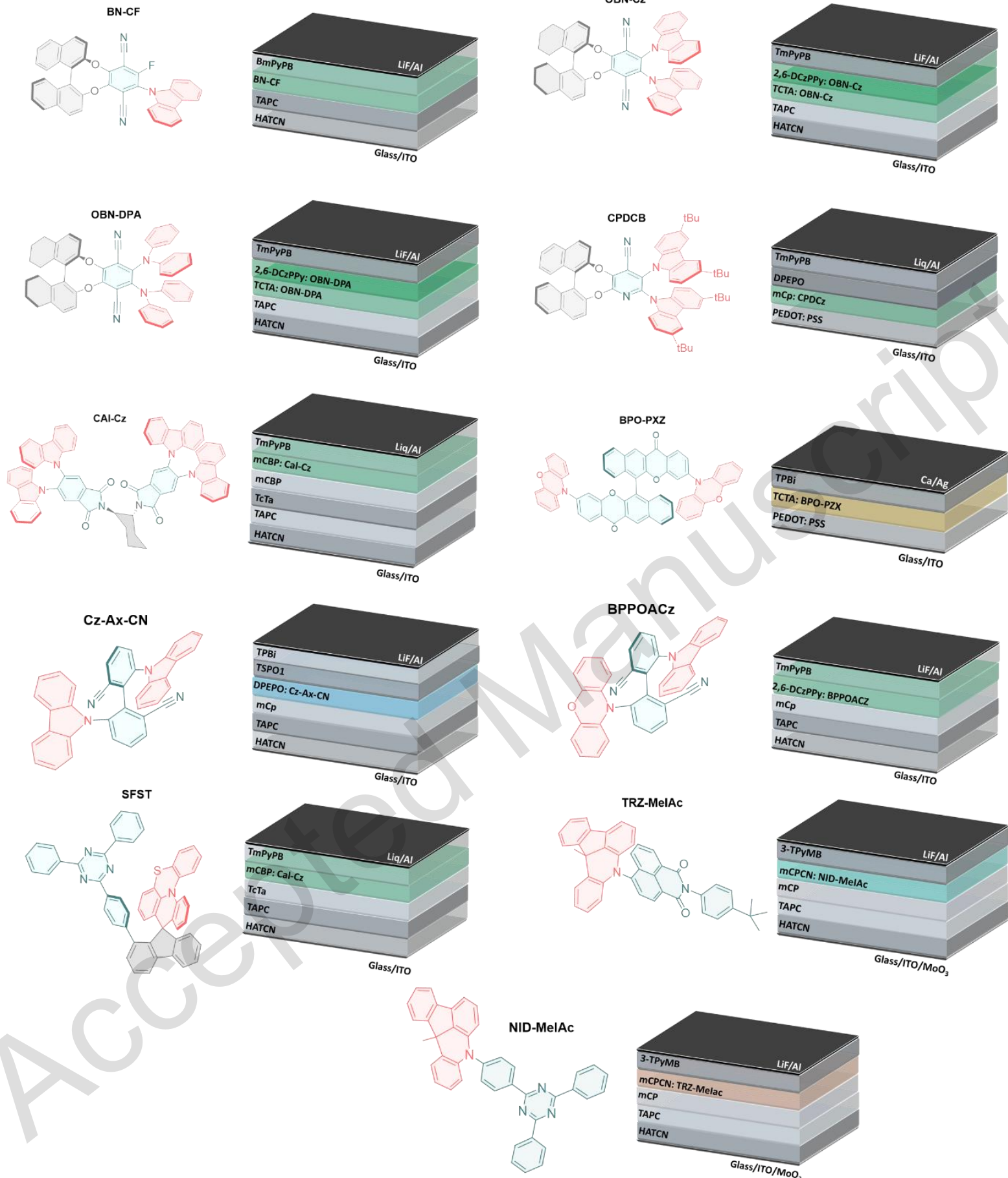

TRZ-MelAc
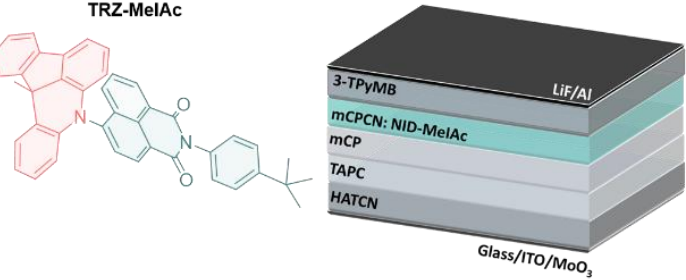

Top-Emission CP-OLED

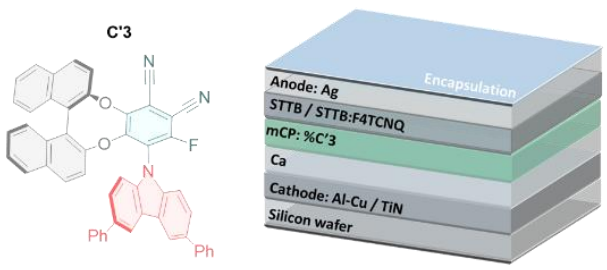

Figure 12: Stack compositions of the CP-OLEDs using CP-TADF molecules 


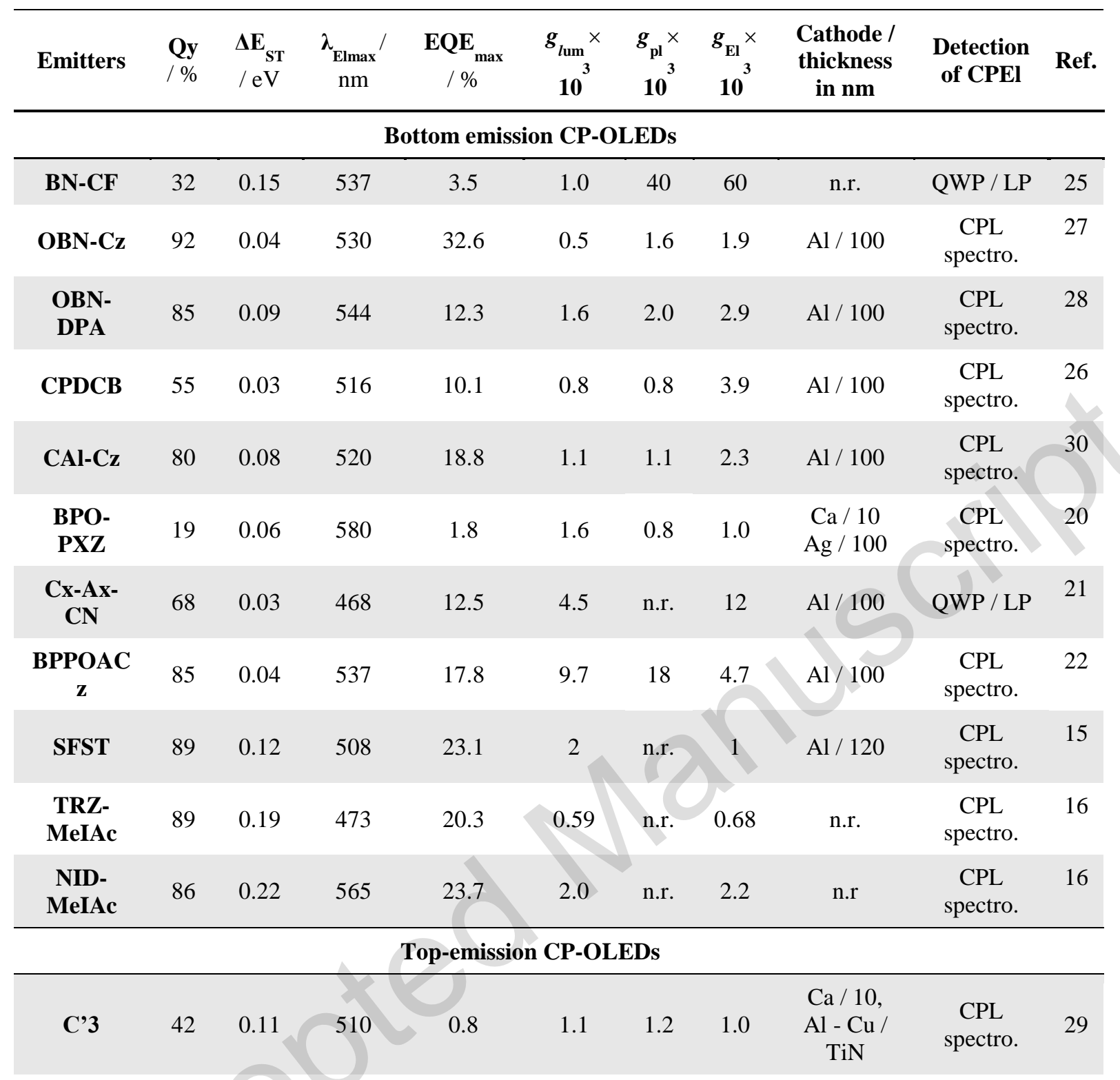

Table 1: Relevant photophysical and chiroptical properties reported for the CP-TADF emitters implemented in CP-OLEDs, together with the obtained performances, the nature of the cathode and the method of detection used for CPEl.

\subsection{Bottom-Emission architecture:}

When the chiral emitter is used as a dopant, the $g_{\text {El }}$ obtained from the devices should theoretically remain in the same order of magnitude as the one measured in diluted solution, since CP luminescence arises from the same molecular entity (although some preferential molecular reorganization may occur in the solid state). This behavior has been observed for several CP-OLEDs based on BPPOACz, SFST and BPO-PXZ as dopant. For these examples, the $g_{\mathrm{El}}$ appears even slightly inferior to the $g_{\text {lum }}$ measured in solution, which may be explained by some depolarization effects arising from device architecture (vide infra). Interestingly, such 
a direct relation between the dissymmetry factors for luminescence and electroluminescence is not always verified since CP-OLEDs including OBN-Cz, CAl-Cz, CPBDCB and OBN-DPA as emitters exhibit an increase of the $g_{\mathrm{El}}$, with more than a factor 4 in the case of CPBDCB. For OBN-Cz, the authors also reported an enhanced $g_{\text {lum }}$ factor both in neat and doped films, probably arising from a preferential molecular conformation in the solid state beneficial to the chiroptical properties. Regarding CPBDCB, the enhancement of circularly polarized electroluminescence was not related to the same effect since the measured CPL values for neat films were in the same order of magnitude as the ones obtained in diluted solution ( $g_{\text {neat film } ~}$ $3.5 \times 10^{-4}$ ), and as in the CP-OLEDs made by vacuum-deposition process $\left(g_{\mathrm{El}}\right.$ of ca. $\left.7 \times 10^{-4}\right)$. The authors ascribed this surprising increase to the chiral molecules being oriented by the centrifugal force during spin-coating deposition of the active layer but no further experiments were performed to corroborate this assertion. ${ }^{[26]}$ While the CP-OLEDs described so far show $g_{\mathrm{El}}$ in the range of $10^{-3}$, consistent with the $g_{\text {lum }}$ observed in solution, two recent device examples have reached impressive $g_{\mathrm{El}}$ values in the order of $10^{-1}-10^{-2}$. Tang et al. were the first to report an unprecedented $g_{\mathrm{El}}$ of $-9.1 \times 10^{-2}$ for CP-OLEDs based on chiral TADF emitters, structurally close to the molecular design developed by some of us, which has also been investigated by the group of Zheng. As explained earlier, while in diluted conditions the compounds showed $\left|g_{\text {lum }}\right|$ around $10^{-3}$, these factors dramatically increased up to $4.2 \times 10^{-2}$ for neat films, due to chiroptical amplification of the excited state attributed to the formation of chiral aggregates in the solid state. Surprisingly, the CP-OLED using BN-CF dispersed in an $m \mathrm{CP}$ host matrix as the emissive layer afforded a $g_{\mathrm{El}}$ of $2.6 \times 10^{-2}$, despite the lack of chiral aggregates, and $6.0 \times 10^{-2}$ for devices based on neat BN-CF as the active layer. Despite being very interesting, this high value of $g_{\mathrm{El}}$, and especially this chiral excited-state amplification, remains the only example of such magnitude and may involve subtle structural and electronic factors since similar molecular designs have not led to the same behaviour. More recently, Chen et al. reported the second example of a CP-OLED with a $g_{\mathrm{El}}$ value exceeding $10^{-2}$, based on the use of $\mathbf{C z}-\mathbf{A x}-\mathbf{C N}$ as the chiral emissive dopant in a DPEPO matrix (20 wt \%). They observed a two-fold increase of the circular polarization in comparison to measurements in diluted solution $\left(\left|g_{\text {lum }}\right|=5.0 \times 10^{-3}\right)$, without further investigation. For these latter two studies, one can note that CPEL has been characterized using the combination of a quarter-wave plate and linear polarizer, while CPL measured in solution has been conducted with a CPL spectrometer. Accordingly, these two obtained values are rather difficult to compare accurately (vide infra).

\subsection{Top-Emission architecture:}


So far, all the research conducted on CP-TADF emitters have resulted in the design of CPOLEDs based on a bottom-emission architecture, i.e. when the emitted CP-light passes through the transparent bottom electrode and substrate on which the architecture has been manufactured. With the aim of investigating other device configurations and bringing new opportunities for chiral emitter applications, we have recently turned our attention to top-emission CP-OLEDs, which refers to an architecture where the $\mathrm{CP}$-light is emitted through the material (cathode, encapsulation layer) that is added following device fabrication (Figure 12). In addition to its potential of achieving higher brightness than the bottom-emission configuration, this type of architecture presents the particularity to be implemented in current microelectronic technologies based on silicon wafer processing, which allows the fabrication of micro-OLEDs with high resolution, strongly relevant for micro-display applications such as cameras, near-eye displays and medical analysis. Surprisingly, top-emission OLEDs have remained unexplored in the context of CP-OLED, despite being a new approach to investigate the impact of device architecture on the propagation of CP-light and its possible depolarization through reflection at the metallic electrode. Indeed, the electroluminescent light in a top-emission device architecture is subjected to various multi-reflections inside the OLED stack, making the control of emitted light polarization particularly challenging. We decided to tackle the challenge of designing the first top-emission CP-OLEDs using optimized CP-TADF emitters based on the rational structure-property study described above. ${ }^{[29]}$ The devices were produced through vacuumdeposition of the chiral dopant in a $m \mathrm{CP}$ matrix, within the following stack: a silicon wafer, covered with an Al-Cu bottom cathode, a thin passivation layer (TiN), a thin layer of calcium (Ca), different electron and hole injection, transport, and blocking layers (EIL/ETL, HTL/HBL), the chiral emissive layer as a dopant ( 15-20\%) in a $m \mathrm{CP}$ matrix and a top ultra-thin silver (Ag) anode, all being encapsulated using a $\mathrm{SiO} / \mathrm{Al}_{2} \mathrm{O}_{3}$ bilayer (Figure 12). While these proof-ofconcept devices have afforded modest performances in terms of current efficiency and EQE, respectively $2.5 \mathrm{~cd} / \mathrm{A}$ and $0.8 \%$, the degree of electroluminescence polarization has been highly preserved, $\left(\left|\mathrm{g}_{\mathrm{El}}\right|=1.0 \times 10^{-3}\right)$, in comparison to the luminescence one measured in diluted solution and neat films, $\left(\left|g_{\text {lum }}\right|=1.1 \times 10^{-3}\right)$, unambiguously demonstrating the possibility of CP-light generation using a top-emission OLED architecture. Importantly, the optical resonance effect used to enhance colour purity and improve light-emission efficiency in the micro-cavity structure seems not detrimental to the generation of polarized electroluminescence and presents new opportunities for the use of CP-TADF in high-resolution micro-display applications. 


\section{Comments and Perspectives}

The design of CP-TADF emitters is undoubtedly a growing area, as illustrated by the rapidly growing number of publications (12 papers in the last 2 years out of a total of 18 since the seminal report in 2015). While several synthetic approaches have been investigated to merge chirality and TADF properties, as described in this progress report, the obtained intensity of $\mathrm{CP}$-Light generated by the reported OLEDs remains too low in view of practical applications. This can be firstly explained by the fact that this research topic is still in its infancy. In this last section, we want to raise some possible perspectives about the design of more efficient $\mathrm{CP}$ TADF emitters and comments that one should take into consideration regarding the elaboration and characterization of devices.

\subsection{Innovative molecular design to reach higher $g_{\text {lum values }}$}

One of the main objectives of chemists working on this research topic is certainly the design of chiral emitters with higher intensity of CPL. To reach this goal, it is crucial to conduct comprehensive research to decipher the key structural and electronic parameters governing the amplitude of CPL, while retaining TADF properties. Recent progress in theoretical chemistry has allowed a deeper understanding of such parameters by granting access to the relative orientation and magnitude of the electric and magnetic transition dipole moments related to the emission process. This should help synthetic chemists conduct structure-property relationships studies and identify the requisite molecular factors to ensure higher CPL intensity. It also appears interesting to take inspiration from the recent results obtained by non-TADF CPL emitters, which display much higher $g_{\text {lum }}$ values, up 5-6 $\times 10^{-2}$, at the molecular level. ${ }^{[11]}$ Regarding this aspect, the difficulty is probably to combine such intense CPL with TADF properties. A promising direction towards this objective may be the development of innovative chiral TADF polymers, and intermolecular concepts such as triplet-triplet upconversion, ${ }^{[34]}$ and $\mathrm{CP}$ resonance energy transfer ${ }^{[35]}$ involving chiral molecules, which have recently shown interesting results in terms of CPL intensity. Likewise, the use of CPL fluorescent emitters, displaying a high dissymmetry factor ${ }^{[36]}$ (and narrow emission spectrum for increased colour purity), embedded in a TADF host matrix, represents an interesting future field of investigation.

\subsection{Further devices considerations}


Because of the complexity of chiroptical measurements in the solid or thin film states, careful and systematic comparison of $g_{\text {lum }}$ values in solution or in thin film with the $g_{\mathrm{El}}$ of OLEDs remains essential, the latter being subject to possible measurement artefacts.

In light of all the results obtained so far for CP-OLEDs, we want to raise in this last section some fundamental questions that, in our opinion, still need to be investigated when moving from measured CPL in diluted solution to the detection of CP-electroluminescence in OLEDs. Firstly, it is important to note that that the use of a quarter-wave plate and a linear polarizer between the CP-OLED and the detector must be employed with caution since the sensitivity of this type of system is rather low and therefore may not be appropriate for classical chiral organic $\left|g_{\text {lum }}\right|$ values (in the order of $\left.10^{-3}\right) \cdot{ }^{[37]}$ For devices based on chiral TADF emitters, the use of a CPL spectrometer is thus preferable since it relies on an electro-optical or photoelastic light modulator, showing higher sensitivity to CP-light and allowing an accurate comparison of literature results.

Secondly, CP-light obtained from OLEDs arises after excitons formation within the active layer and diffusion through several solid layers sandwiched between conductive electrodes, one of which is metallic.

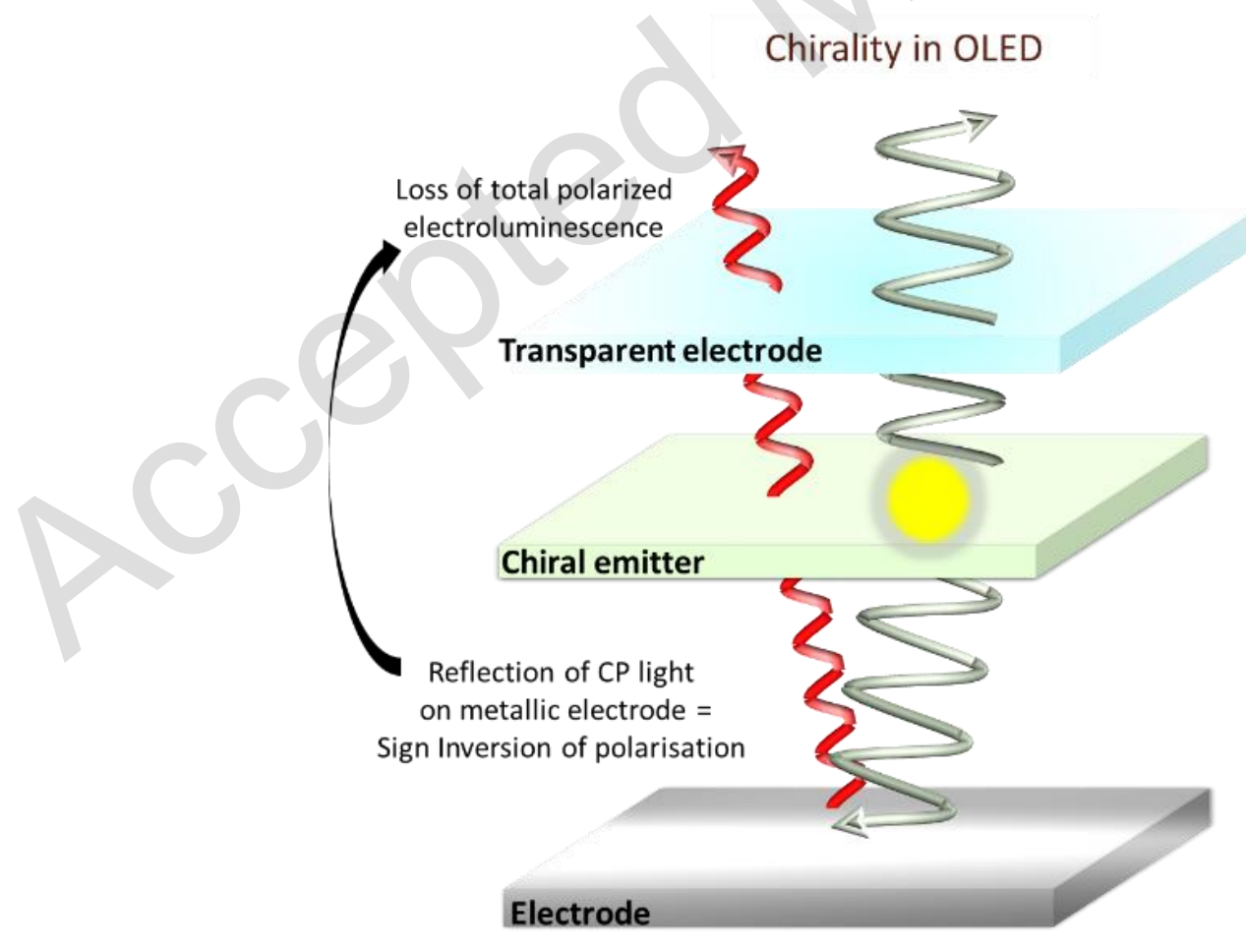

Figure 13: Schematic illustration of the reflection of generated CP light from the chiral emitter (arrows in light green) at the metal electrode, reducing the total polarization of the outgoing electroluminescence 
In classical emitting device architectures, the electro-generated light is extracted from the device through a transparent anode, often based on indium tin oxide, ITO. At the other side of the device, the cathode is therefore rendered highly reflective, for instance using an aluminium layer, to maximize the intensity of the outgoing light at the transparent ITO layer. While this aspect is beneficial for OLED brightness, it is however detrimental to the polarization of electroluminescence since the reflection reverses the handedness of CP light (Figure 13). In fact, once charge recombination happens, the resulting emission of the chiral emitter occurs towards both electrodes from the active layer and thus a significant part of this polarized luminescence may reach the metallic one, resulting in a sign inversion of circular polarization upon reflection and a loss of total CP electroluminescence intensity in comparison to the degree of CPL obtained for the chiral emitter in solution. This crucial aspect remains unexplored for $\mathrm{CP}$ OLEDs based on TADF emitters, despite its first mention for devices based on chiral organic polymeric materials by Dekkers and Meijer, ${ }^{[38]}$ and its clear evidence reported by Di Bari et al., ${ }^{[39], ~[40] ~ f o r ~ C P-O L E D s ~ u s i n g ~ c h i r a l ~ l a n t h a n i d e ~ c o m p l e x e s . ~ I n d e e d, ~ t h e ~ l a t t e r ~ a u t h o r s ~}$ circumvented this problem by moving to a barium/aluminum cathode with a very low thickness of $12 \mathrm{~nm}$, resulting in a semi-transparent metallic electrode with lower reflection efficiency. Interestingly, this potential source of reflection/depolarization does not seem to affect more recent CP-OLEDs based on polymeric emitters, or molecular emitters embedded in a polymeric matrix, ${ }^{[41],[42]}$ reinforcing the need for further exploration of device architecture effects when developing CP-OLEDs with chiral TADF emitters, as it has been done for other classes of CPL

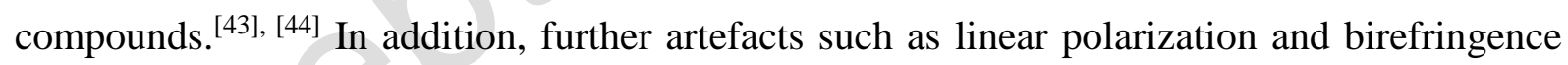
from other components of the device architecture can lead to discrepancies in the measured values of CP electroluminescence, as well as the position of the charge recombination zone. ${ }^{[43] \text {, }}$ [44], [45] Because these phenomena are not always easy to detect and analyze, it may be worth developing analytical models such as Müeller matrix analysis to gain insights into these deleterious aspects. Finally, it also seems important to take into consideration the possible degradations of the chiral emitter within the device, both chemically and configurationally. As far as the authors know, such investigations are currently unexplored in this context, albeit being of crucial importance, especially with regards to the intensity of CPEL. Dedicated device engineering will be also crucial to increase the performances of CP-OLEDs both in terms of luminance and dissymmetry factor. In this context, studies regarding the influence of the device architectures (nature, thickness, order of stacking of the different layers) and the development 
of cathodes with low reflectivity to limit sign inversion of the CPEL represent interesting avenues.

\section{Conclusion}

This progress report recounts 5 years of international research activities devoted to the development of small organic chiral fluorophores combining TADF and CPL emission properties. Although these display promising properties, a significant increase of the dissymmetry factor (by one or two orders of magnitude) is still a requirement in order to unlock the potential of these materials for concrete applications and show substantial benefits to using these chiral molecules rather than their achiral counterparts in OLEDs. To reach this goal, it is crucial to conduct rigorous research to decipher the key of both structural and electronic parameters governing the amplitude of CPL, while retaining TADF properties. Recent progress in theoretical chemistry has allowed a deeper understanding of such parameters by granting access to the relative orientation and magnitude of the electrical and magnetic transition dipole moments in the exited state. Because of the complexity of chiroptical measurements in the solid

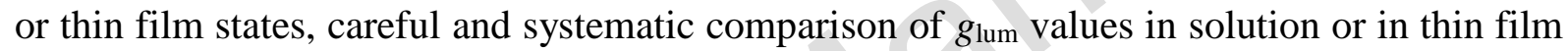
with and the $g_{\mathrm{El}}$ of OLEDs remains essential, the latter being subject to possible measurement artefacts. In order to reach higher dissymmetry factors, investigating controlled supramolecular assemblies permitting an amplification of the CPL signal without affecting photophysical performance appears as a cornerstone approach to provide real-world benefits over nonpolarized OLEDs. ${ }^{[46]}$ Alongside the search for more efficient CP-TADF molecular designs, device engineering is also of paramount importance to limit depolarization induced by light reflection in the OLED stack, and particularly at the cathode commonly made of a reflective material such as aluminum. Triplet-triplet upconversion, ${ }^{[34]}$ and $\mathrm{CP}$ resonance energy transfer ${ }^{[35]}$ involving chiral molecules are also promising avenues for further developments. Likewise, the use of CPL fluorescent emitters, displaying a high dissymmetry factor ${ }^{[36]}$, embedded in a TADF host matrix, represents an interesting future field of investigation. Because of the complexity behind the CP-electroluminescence phenomenon, a close collaboration between organic chemists and physicists appears essential to fully understand and control the different aspects allowing the construction of OLED devices displaying high EQE and dissymmetry factors.

\section{Acknowledgements}


GP, AL and LF thank the SCBM, the Labex CHARMMMAT (ANR-11-LABX-0039) and the "Programme Transverse de Competences du CEA" (POLEM project). LFa acknowledges the Centre National de la Recherche Scientifique (CNRS) and all the authors warmly than the ANR (iChiralight project) for funding and support.

Received: ((will be filled in by the editorial staff))

Revised: ((will be filled in by the editorial staff)) Published online: ((will be filled in by the editorial staff))

\section{References}

[1] A. Salehi, X. Fu, D.-H. Shin, F. So, Adv. Funct. Mater. 2019, 29, 1808803.

[2] M. A. Baldo, M. E. Thompson, S. R. Forrest, Nature 2000, 403, 750-753.

[3] H. Uoyama, K. Goushi, K. Shizu, H. Nomura, C. Adachi, Nature 2012, 492, 234-238.

[4] a) Z. Yang, Z. Mao, Z. Xie, Y. Zhang, S. Liu, J. Zhao, J. Xu, Z. Chi, M. P. Aldred,

Chem. Soc. Rev. 2017, 46, 915-1016 b) M. Y. Wong, E. Zysman-Colman, Adv. Mater. 2017, 29, 1605444 c) Godumala, M.; Choi, S.; Cho, M. J.; Choi, D. H. J. Mater. Chem. C 2019, 7, 2172-2198 d) Yin, X.; He, Y.; Wang, X.; Wu, Z.; Pang, E.; Xu, J.; Wang, J.-a. Front. Chem. 2020, 8, 725. e) Liang, X.; Tu, Z.-L.; Zheng, Y.-X. Chem. Eur. J. 2019, 25, 5623-5642.

[5] Madayanad Suresh, S.; Hall, D.; Beljonne, D.; Olivier, Y.; Zysman-Colman, E. Adv. Funct. Mater. 2020, 30, 1908677.

[6] J.-M. Teng, Y.-F. Wang, C.-F. Chen, J. Mater. Chem. C 2020, 8, 11340-11353.

[7] D.-W. Zhang, M. Li, C.-F. Chen, Chem. Soc. Rev. 2020, 49, 1331-1343.

[8] F. Zinna, L. Di Bari, Chirality 2015, 27, 1-13. 
[9] J. R. Brandt, F. Salerno, M. J. Fuchter, Nat. Rev. Chem. 2017, 1, 0045.

[10] E. M. Sánchez-Carnerero, A. R. Agarrabeitia, F. Moreno, B. L. Maroto, G. Muller, M. J. Ortiz, S. de la Moya, Chem. Eur. J. 2015, 21, 13488-13500.

[11] P. Reine, A. G. Campaña, L. Alvarez de Cienfuegos, V. Blanco, S. Abbate, A. J. Mota, G. Longhi, D. Miguel, J. M. Cuerva, Chem. Commun. 2019, 55, 10685-10688.

[12] Zhou, L.; Xie, G.; Ni, F.; Yang, C. Appl. Phys. Lett. 2020, 117, 130502.

[13] T. Imagawa, S. Hirata, K. Totani, T. Watanabe, M. Vacha, Chem. Commun. 2015, 51, 13268-13271.

[14] F.-Y. Hao, Y.-Z. Shi, K. Wang, S.-Y. Xiong, X.-C. Fan, L. Wu, C.-J. Zheng, Y.-Q. Li, X.-M. Ou, X.-H. Zhang, Dyes Pigments 2020, 178, 108336.

[15] S.-Y. Yang, Y.-K. Wang, C.-C. Peng, Z.-G. Wu, S. Yuan, Y.-J. Yu, H. Li, T.-T.

Wang, H.-C. Li, Y.-X. Zheng, Z.-Q. Jiang, L.-S. Liao, J. Am. Chem. Soc. 2020, 142, 1775617765.

[16] F. Ni, C.-W. Huang, Y. Tang, Z. Chen, Y. Wu, S. Xia, X. Cao, J.-H. Hsu, W.-K. Lee, K. Zheng, Z. Huang, C.-C. Wu, C. Yang, Mater. Horiz. 2021.

[17] Z.-Y. Gu, C.-G. Liu, S.-Y. Wang, S.-J. Ji, Org. Lett. 2016, 18, 2379-2382.

[18] M.-Y. Zhang, Z.-Y. Li, B. Lu, Y. Wang, Y.-D. Ma, C.-H. Zhao, Org. Lett. 2018, 20, $6868-6871$.

[19] N. Sharma, E. Spuling, C. M. Mattern, W. Li, O. Fuhr, Y. Tsuchiya, C. Adachi, S. Bräse, I. D. W. Samuel, E. Zysman-Colman, Chem. Sci. 2019, 10, 6689-6696.

[20] Y. Wang, Y. Zhang, W. Hu, Y. Quan, Y. Li, Y. Cheng, ACS Appl. Mater. Interfaces 2019, 11, 26165-26173.

[21] M. Li, Y.-F. Wang, D. Zhang, L. Duan, C.-F. Chen, Angew. Chem. Int. Ed. 2020, 59, 3500-3504.

[22] Z.-L. Tu, Z.-P. Yan, X. Liang, L. Chen, Z.-G. Wu, Y. Wang, Y.-X. Zheng, J.-L. Zuo, Y. Pan, Adv. Sci. 2020, 7, 2000804. 
[23] Y.-F. Wang, M. Li, W.-L. Zhao, Y.-F. Shen, H.-Y. Lu, C.-F. Chen, Chem. Commun. 2020, 56, 9380-9383.

[24] S. Feuillastre, M. Pauton, L. Gao, A. Desmarchelier, A. J. Riives, D. Prim, D. Tondelier, B. Geffroy, G. Muller, G. Clavier, G. Pieters, J. Am. Chem. Soc. 2016, 138, 39903993.

[25] F. Song, Z. Xu, Q. Zhang, Z. Zhao, H. Zhang, W. Zhao, Z. Qiu, C. Qi, H. Zhang, H. H. Y. Sung, I. D. Williams, J. W. Y. Lam, Z. Zhao, A. Qin, D. Ma, B. Z. Tang, Adv. Funct. Mater. 2018, 28, 1800051.

[26] S. Sun, J. Wang, L. Chen, R. Chen, J. Jin, C. Chen, S. Chen, G. Xie, C. Zheng, W. Huang, J. Mater. Chem. C 2019, 7, 14511-14516.

[27] Z.-G. Wu, H.-B. Han, Z.-P. Yan, X.-F. Luo, Y. Wang, Y.-X. Zheng, J.-L. Zuo, Y. Pan, Adv. Mater. 2019, 31, 1900524.

[28] Z.-G. Wu, Z.-P. Yan, X.-F. Luo, L. Yuan, W.-Q. Liang, Y. Wang, Y.-X. Zheng, J.-L. Zuo, Y. Pan, J. Mater. Chem. C 2019, 7, 7045-7052.

[29] L. Frédéric, A. Desmarchelier, R. Plais, L. Lavnevich, G. Muller, C. Villafuerte, G. Clavier, E. Quesnel, B. Racine, S. Meunier-Della-Gatta, J.-P. Dognon, P. Thuéry, J. Crassous, L. Favereau, G. Pieters, Adv. Funct. Mater. 2020, 30, 2004838.

[30] M. Li, S.-H. Li, D. Zhang, M. Cai, L. Duan, M.-K. Fung, C.-F. Chen, Angew. Chem. Int. Ed. 2018, 57, 2889-2893.

[31] M. Li, Y. Liu, R. Duan, X. Wei, Y. Yi, Y. Wang, C.-F. Chen, Angew. Chem. Int. Ed. 2017, 56, 8818-8822.

[32] a) Szymkowiak, J.; Kwit, M. Chirality 2018, 30, 117-130. b) Berova, N.; Bari, L. D.; Pescitelli, G. Chem. Soc. Rev. 2007, 36, 914-931. c) Harada, N., Nakanishi, K. and Berova, N. (2012). Electronic CD Exciton Chirality Method: Principles and Applications. In Comprehensive Chiroptical Spectroscopy (eds N. Berova, P.L. Polavarapu, K. Nakanishi and 
R.W. Woody). d) Gawroński, J.; Brzostowska, M.; Kacprzak, K.; Kołbon, H.; Skowronek, P. Chirality 2000, 12, 263-268.

[33] Y.-F. Wang, H.-Y. Lu, C. Chen, M. Li, C.-F. Chen, Org. Electron. 2019, 70, 71-77.

[34] Ieuji, R.; Goushi, K.; Adachi, C. Nat. Commun. 2019, 10, 5283.

[35] Wade, J.; Brandt, J.; Reger, D.; Zinna, F.; Amsharov, K.; Jux, N.; Andrews, D.;

Fuchter, M. J. Angew. Chem. Int. Ed. 2020, n/a.

[36] Li, M.; Wang, Y.-F.; Zhang, D.-W.; Zhang, D.; Hu, Z.-Q.; Duan, L.; Chen, C.-F. Sci. China Mater. 2020.

[37] G. Longhi, E. Castiglioni, J. Koshoubu, G. Mazzeo, S. Abbate, Chirality 2016, 28, 696-707.

[38] E. Peeters, M. P. T. Christiaans, R. A. J. Janssen, H. F. M. Schoo, H. P. J. M. Dekkers, E. W. Meijer, J. Am. Chem. Soc. 1997, 119, 9909-9910.

[39] F. Zinna, U. Giovanella, L. D. Bari, Adv. Mater. 2015, 27, 1791-1795.

[40] F. Zinna, M. Pasini, F. Galeotti, C. Botta, L. Di Bari, U. Giovanella, Adv. Funct. Mater. 2017, 27, 1603719.

[41] J. R. Brandt, X. Wang, Y. Yang, A. J. Campbell, M. J. Fuchter, J. Am. Chem. Soc. 2016, 138, 9743-9746.

[42] D. Di Nuzzo, C. Kulkarni, B. Zhao, E. Smolinsky, F. Tassinari, S. C. J. Meskers, R. Naaman, E. W. Meijer, R. H. Friend, ACS Nano 2017, 11, 12713-12722.

[43] L. Wan, J. Wade, F. Salerno, O. Arteaga, B. Laidlaw, X. Wang, T. Penfold, M. J. Fuchter, A. J. Campbell, ACS Nano 2019, 13, 8099-8105.

[44] L. Wan, J. Wade, X. Shi, S. Xu, M. J. Fuchter, A. J. Campbell, ACS Appl. Mater. Interfaces 2020, 12, 39471-39478.

[45] J.-H. Jung, D.-M. Lee, J.-H. Kim, C.-J. Yu, J. Mater. Chem. C 2018, 6, 726-730.

[46] a)Nitti, A.; Pasini, D. Adv. Mater. 2020, 32, 1908021. b) Sang, Y.; Han, J.; Zhao, T.; Duan, P.; Liu, M. Adv. Mater. 2020, 32, 1900110. 


\section{Biographies:}

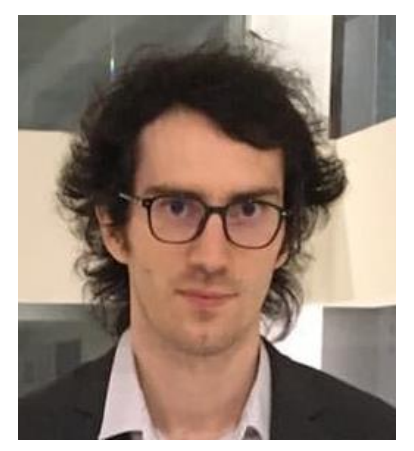

Lucas Frédéric received his Engineer degree in Chemistry and Chemical Engineering in 2017 from the National Institute of Applied Sciences (INSA) in Rouen. In parallel, he obtained a master degree in organic chemistry from University of Rouen. He performed his Ph.D. under the supervision of Dr. Grégory Pieters at CEA Saclay on synthesis and characterization of new circularly polarized electroluminescent molecules; he received his degree from University Paris-Saclay in October 2020. He moved then as a post-doctoral fellow in the University of Geneva with Prof. Jérôme Lacour where he is currently working on synthesis of cationic helicenes.

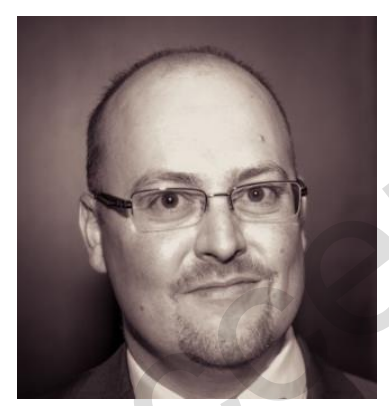

Alaric Desmarchelier received his master's degree in organic chemistry in 2009 from the University of Versailles (F), and his $\mathrm{PhD}$ from the same institution in 2012 under the guidance of Profs. C. Greck and X. Moreau, studying organocatalytic tandem/cascade reactions. After a first postdoctoral fellowship at the University of Groningen (NL) in asymmetric coppercatalyzed transformations with Prof. S. Harutyunyan, he worked with Drs L. Bouteiller and M. Raynal at UPMC Paris 6 university (F) on chiral supramolecular self-assembling catalysts, in close collaboration with Profs. Piet Van Leeuwen and Anton Vidal at ICIQ (E). He then joined Dr Pieters' team at CEA Saclay (F) to develop new CP-TADF fluorophores and study their applications in OLEDs. Following a stay at Ecole Polytechnique and ICSN-CNRS (F) with Drs. 
Abderrahim Yassar and Philippe Dauban focusing on polyaromatic semi-conductors and their late-stage functionalization, he moved to Scotland where he currently works as a Senior Scientist in pharmaceutical process development at Almac Sciences in Edinburgh.

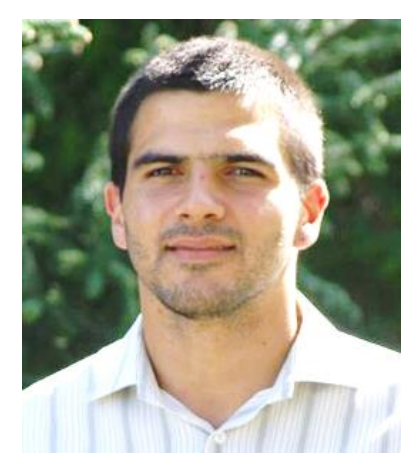

Ludovic Favereau received his master's degree in organic chemistry in 2011 from the National Institute of Applied Sciences (INSA) in Rouen. He obtained his Ph.D. in 2014 from the University of Nantes under the supervision of Dr. F. Odobel on the synthesis of molecular architectures for mimicking the photosynthetic $\mathrm{Z}$ scheme function. After a one-year postdoctoral fellow at the University of Oxford with Prof. Harry L. Anderson on the synthesis of porphyrin nanorings, he was recruited as CNRS researcher at the Institut des Sciences Chimiques de Rennes (University of Rennes, France) in 2015 in the organometallic: materials and catalysis (OMC) group with Dr J. Crassous. In 2019, he received the Marc Julia prize from the Société Chimique de France. His research focuses on the design of chiral organic closedand open-shell $\pi$-conjugated molecules with intense chiroptical properties (circular dichroism, circularly polarized luminescence) and chiral radicals to explore the potential of chirality in material science (OLED, OPV, ...).

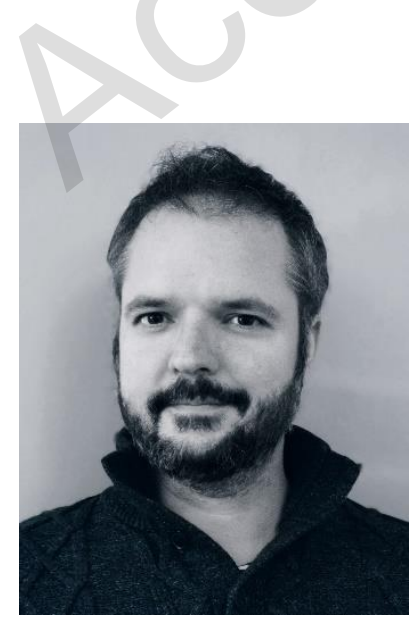


Grégory Pieters received his master's degree in organic chemistry in 2006 from the Université Pierre et Marie Curie (UPMC) in Paris. He obtained his Ph.D. in 2010 from the University of Versailles-Saint- Quentin-en-Yvelines under the supervision of Dr. D. Prim on the synthesis of functionalized polyaromatic molecules. After a one-year and a half postdoctoral fellow at the University of Padova with Prof. L. J. Prins on the assembly of oliganionic molecules around functionalized gold nanoparticles, he was recruited as CEA researcher in 2013 at the Service de Chimie Bioorganique et de Marquage (CEA Paris Saclay) with Dr. B. Rousseau. In 2017, he obtained his Habilitation (HDR) and became leader of the tritium-labelling group. His research focuses on the development of Hydrogen Isotope Exchange reactions and the design of chiral fluorophores. 